\title{
Hochschild cohomology, the characteristic morphism and derived deformations
}

\author{
Wendy Lowen
}

\begin{abstract}
A notion of Hochschild cohomology $H H^{*}(\mathcal{A})$ of an abelian category $\mathcal{A}$ was defined by Lowen and Van den Bergh (Adv. Math. 198 (2005), 172-221). They also showed the existence of a characteristic morphism $\chi$ from the Hochschild cohomology of $\mathcal{A}$ into the graded centre $\mathfrak{Z}^{*}\left(D^{b}(\mathcal{A})\right)$ of the bounded derived category of $\mathcal{A}$. An element $c \in H H^{2}(\mathcal{A})$ corresponds to a first-order deformation $\mathcal{A}_{c}$ of $\mathcal{A}$ (Lowen and Van den Bergh, Trans. Amer. Math. Soc. 358 (2006), 5441-5483). The problem of deforming an object $M \in D^{b}(\mathcal{A})$ to $D^{b}\left(\mathcal{A}_{c}\right)$ was treated by Lowen (Comm. Algebra 33 (2005), 3195-3223). In this paper we show that the element $\chi(c)_{M} \in \operatorname{Ext}_{\mathcal{A}}^{2}(M, M)$ is precisely the obstruction to deforming $M$ to $D^{b}\left(\mathcal{A}_{c}\right)$. Hence, this paper provides a missing link between the above works. Finally we discuss some implications of these facts in the direction of a 'derived deformation theory'.
\end{abstract}

\section{Introduction}

Let $k$ be a commutative ring. It is well known that for a $k$-algebra $A$, there is a characteristic morphism $\chi_{A}$ of graded commutative algebras from the Hochschild cohomology of $A$ to the graded centre of the derived category $D(A)$. If $k$ is a field, this morphism is determined by the maps, for $M \in D(A)$,

$$
M \otimes_{A}^{L}-: H H_{k}^{*}(A) \cong \operatorname{Ext}_{A^{\text {op }}}^{*} \otimes_{k} A(A, A) \longrightarrow \operatorname{Ext}_{A}^{*}(M, M) .
$$

The characteristic morphism plays an important role, for example, in the theory of support varieties [AB00, EHTSS04, SS04]. Characteristic morphisms were generalized to various situations where a good notion of Hochschild cohomology is at hand. Recently, Buchweitz and Flenner defined and studied Hochschild cohomology for morphisms of schemes or analytic spaces, and proved the existence of a characteristic morphism in this context [BF08]. In [Kel03], Keller defined the Hochschild cohomology of an exact category as the Hochschild cohomology of a certain dg quotient. For an abelian category $\mathcal{A}$, this is precisely the Hochschild cohomology of a 'dg enhancement' of the bounded derived category $D^{b}(\mathcal{A})$. Consequently, the projection on the zero part of the Hochschild complex (see $\S 2.5$ ) is itself a natural dg enhancement of a characteristic morphism

$$
\chi_{\mathcal{A}}: H H_{\mathrm{ex}}^{*}(\mathcal{A}) \longrightarrow \mathfrak{Z}^{*}\left(D^{b}(\mathcal{A})\right),
$$

where the right-hand side denotes the graded centre of $D^{b}(\mathcal{A})$ (see $\left.\S 4.2\right)$. Explicitly, $\chi_{\mathcal{A}}$ maps a Hochschild $n$-cocycle $c$ to a collection of elements $\chi_{\mathcal{A}}(c)_{M} \in \operatorname{Ext}_{\mathcal{A}}^{*}(M, M)$ for $M \in D^{b}(\mathcal{A})$. The main purpose of this paper is to give an interpretation of $\chi_{\mathcal{A}}(c)_{M}$ in terms of deformation theory.

Received 16 July 2007, accepted in final form 25 March 2008.

2000 Mathematics Subject Classification 18G60 (primary), 13D10 (secondary).

Keywords: Hochschild cohomology, $B_{\infty}$-algebra, characteristic morphism, deformation, abelian category, derived category, $A_{[0, \infty[\text {-category. }}$

The author is a postdoctoral fellow with FWO/CNRS.

This journal is (C) Foundation Compositio Mathematica 2008. 


\section{W. LOWEN}

In [LV06], a deformation theory of abelian categories was developed. Its relation with Hochschild cohomology goes through an alternative definition of the latter given by the authors in [LV05], and shown in the same paper to be equivalent to Keller's definition. Let us consider, from now on, an abelian category $\mathcal{A}$ with enough injectives, and let us assume that $k$ is a field. $\operatorname{Then} \operatorname{Inj}(\mathcal{A})$ is a $k$-linear category and we put

$$
H H_{\mathrm{ab}}^{*}(\mathcal{A})=H H^{*}(\operatorname{lnj}(\mathcal{A})) .
$$

The main advantage of $\operatorname{lnj}(\mathcal{A})$ is that, considering it as a ring with several objects, its deformation theory is entirely understood in the sense of Gerstenhaber's deformation theory of algebras [Ger64]. It is shown in [LV06] that the abelian deformation theory of $\mathcal{A}$ is equivalent to the linear deformation theory of $\operatorname{Inj}(\mathcal{A})$, justifying (1). An abelian deformation $\mathcal{B}$ of $\mathcal{A}$ gives rise to a morphism

$$
D^{b}(\mathcal{B}) \longrightarrow D^{b}(\mathcal{A})
$$

and an obstruction theory for deforming objects $M \in D^{b}(\mathcal{A})$ to $D^{b}(\mathcal{B})$, which is the subject of [Low05]. The main theorem of the current paper (see also Theorem 4.8) can be stated as follows.

Theorem 1.1. Consider $c \in H H_{\mathrm{ex}}^{2}(\mathcal{A})$ and let $\mathcal{A}_{c}$ be the corresponding (first-order) deformation of $\mathcal{A}$. For $M \in D^{b}(\mathcal{A})$, the element $\chi_{\mathcal{A}}(c)_{M} \in \operatorname{Ext}_{\mathcal{A}}^{2}(M, M)$ is the obstruction against deforming $M$ to an object of $D^{b}\left(\mathcal{A}_{c}\right)$.

Hence, the characteristic morphism $\chi_{\mathcal{A}}$ is a natural ingredient in a theory describing the simultaneous deformations of an abelian category together with (families or diagrams of) objects in the abelian (or derived) category. The details of this theory remain to be worked out.

In [Low05], (2) is expressed in terms of complexes of injectives in $\mathcal{A}$ and $\mathcal{B}$, and the obstructions are expressed in terms of the element $c \in H H_{\mathrm{ab}}^{2}(\mathcal{A})=H H^{2}(\operatorname{Inj}(\mathcal{A}))$ corresponding to the abelian deformation. Essentially, our approach for proving the above theorem is to tighten the relation between $H H_{\mathrm{ab}}^{*}(\mathcal{A})$ and $H H_{\mathrm{ex}}^{*}(\mathcal{A})$.

For a differential graded category $\mathfrak{a}$, let $\mathbf{C}(\mathfrak{a})$ denote its Hochschild complex [Kel03]. Let $D_{\mathrm{dg}}^{b}(\mathcal{A})$ be a dg model of $D^{b}(\mathcal{A})$ constructed using complexes of injectives. The natural inclusion $\operatorname{Inj}(\mathcal{A}) \subset$ $D_{\mathrm{dg}}(\mathcal{A})$ induces a projection morphism

$$
\mathbf{C}\left(D_{\mathrm{dg}}(\mathcal{A})\right) \longrightarrow \mathbf{C}(\operatorname{lnj}(\mathcal{A}))
$$

which is proven in [LV05] to be a quasi-isomorphism of $B_{\infty}$-algebras. The $B_{\infty}$-structure of the Hochschild complexes captures all of the operations relevant to deformation theory, such as the cup product and the Gerstenhaber bracket, but also the more primitive brace operations (see $\S 2.3$ ). In $\S 3$, we explicitly construct a $B_{\infty}$-section

$$
\operatorname{embr}_{\delta}: \mathbf{C}(\operatorname{lnj}(\mathcal{A})) \longrightarrow \mathbf{C}\left(D_{\mathrm{dg}}(\mathcal{A})\right)
$$

of (3) (Theorem 3.22). In the notation, $\delta$ is the element in $\mathbf{C}^{1}\left(D_{\mathrm{dg}}(\mathcal{A})\right)$ determined by the differentials of the complexes of injectives, and embr, short for 'embrace', refers to the brace operations. More concretely, for $c \in \mathbf{C}^{n}(\operatorname{Inj}(\mathcal{A}))$, we have

$$
\operatorname{embr}_{\delta}(c)=\sum_{m=0}^{n} c\left\{\delta^{\otimes m}\right\} .
$$

After introducing the characteristic morphism in $\S 4.3$, we use the morphism embr $_{\delta}$ to prove Theorem 1.1 in $\S 4.4$.

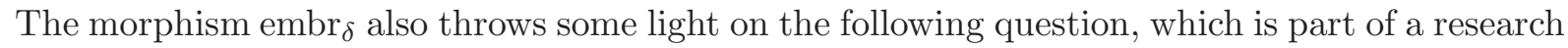
project in progress.

Question. Given an abelian deformation $\mathcal{B}$ of an abelian category $\mathcal{A}$, in what sense can we interpret $D^{b}(\mathcal{B})$ as a 'derived' deformation of $D^{b}(\mathcal{A})$ ? 
More precisely, the morphism $\operatorname{embr}_{\delta}$ gives us a recipe to turn a linear deformation of $\operatorname{Inj}(\mathcal{A})$ (and, hence, an abelian deformation of $\mathcal{A}$ ) into a deformation of $D_{\mathrm{dg}}^{b}(\mathcal{A}) \ldots$ as a $c d g$ category. Here $\mathrm{cdg}$, as opposed to dg, means that apart from compositions $m$ and differentials $d$, the category has 'curvature elements' correcting the fact that $d^{2} \neq 0$. This 'small' alteration has serious consequences, ruining, for example, the notion of quasi-isomorphism.

In Theorem 4.18 we show that the cdg deformation of $D_{\mathrm{dg}}^{b}(\mathcal{A})$ contains a maximal partial dg deformation which is a skeleton of $D_{\mathrm{dg}}^{b}(\mathcal{B})$ (see also Remarks 4.19 and 4.20). The part of $D_{\mathrm{dg}}^{b}(\mathcal{A})$ that is dg deformed in this way is spanned by the 'zero locus' of the characteristic element

$$
\left(M \mapsto \chi_{\mathcal{A}}(c)_{M}\right) \in \prod_{\mathrm{Ob}\left(D^{b}(\mathcal{A})\right)} \operatorname{Ext}_{\mathcal{A}}^{2}(M, M) .
$$

Hence, an object $M \in D^{b}(\mathcal{A})$ contributes to the $\operatorname{dg}$ deformation of $D_{\mathrm{dg}}^{b}(\mathcal{A})$ if and only if it deforms, in the sense of [Low05], to an object of $D^{b}(\mathcal{B})$.

\section{2. $A_{[0, \infty[\text {-categories }}$}

Nowadays $A_{\infty}$-algebras and categories are widely used as algebraic models for triangulated categories (see [BLM06, HL04, Kel06, KS06] and the references therein). Although the generalization to the $A_{[0, \infty}$ [-setting causes serious new issues, a large part of the theory can still be developed 'in the $A_{\infty}$-spirit'. In this section we try to give a brief, reasonably self-contained account of the facts we need. For more detailed accounts we refer the reader to [GJ90, Laz03] for $A_{\infty}$-algebras, to [Lef03, Lyu03] for $A_{\infty}$-categories and to [Nic07] for $A_{[0, \infty}$ [-algebras.

\subsection{A word on signs and shifts}

Let $k$ be a commutative ring. All of the algebraic constructions in this paper take place in and around the category $G(k)$ of $\mathbb{Z}$-graded $k$-modules. For $M, N \in G(k)$, we have the familiar tensor product

$$
(M \otimes N)^{n}=\bigoplus_{i \in \mathbb{Z}} M^{i} \otimes N^{n-i}
$$

and internal hom

$$
[M, N]^{p}=\prod_{i \in \mathbb{Z}} \operatorname{Hom}\left(M^{i}, M^{i+p}\right)
$$

over $k$. For $m \in M^{i}$, the degree of $m$ is $|m|=i$. We adopt the Koszul sign convention, that is, $G(k)$ is endowed with the well-known closed tensor structure with 'super' commutativity isomorphisms

$$
M \otimes N \longrightarrow N \otimes M: m \otimes n \longmapsto(-1)^{|m||n|} n \otimes m
$$

and the standard associativity and identity isomorphisms. The closed structure is determined by the evaluation morphism

$$
[M, N] \otimes M \longrightarrow N:(f, m) \longmapsto f(m)
$$

Furthermore, we make a choice of shift functors on $G(k)$. For $i \in \mathbb{Z}$, let $\Sigma^{i} k \in G(k)$ be the object whose only nonzero component is $\left(\Sigma^{i} k\right)^{-i}=k$. The shift functors are the functors

$$
\Sigma^{i}=\Sigma^{i} k \otimes-: G(k) \longrightarrow G(k): M \longmapsto \Sigma^{i} M=\Sigma^{i} k \otimes M .
$$

For $m \in M$, we put $\sigma^{i} m=1 \otimes m \in \Sigma^{i} M$. All of the canonical isomorphisms (and, in particular, the signs) in this paper are derived from the above conventions. The most general canonical isomorphisms we will use are of the form, for $M_{1}, \ldots, M_{n}, M \in G(k)$ :

$$
\varphi: \Sigma^{i-i_{1}-\cdots-i_{n}}\left[M_{1} \otimes \cdots \otimes M_{n}, M\right] \longrightarrow\left[\Sigma^{i_{1}} M_{1} \otimes \cdots \otimes \Sigma^{i_{n}} M_{n}, \Sigma^{i} M\right]
$$




\section{W. LOWEN}

defined by $\varphi\left(\sigma^{i-i_{1}-\cdots-i_{n}} \phi\right)\left(\sigma^{i_{1}} m_{1}, \ldots, \sigma^{i_{n}} m_{n}\right)=(-1)^{\alpha} \sigma^{i} \phi\left(m_{1}, \ldots, m_{n}\right)$, where

$$
\alpha=\left(i_{1}+\cdots+i_{n}\right)|\phi|+i_{2}\left|m_{1}\right|+\cdots+i_{n}\left(\left|m_{1}\right|+\cdots+\left|m_{n-1}\right|\right) .
$$

\subsection{The Hochschild object of a (graded) quiver}

In this section and the next we introduce the Hochschild complex of an $A_{[0, \infty}$ [-category (see also [GJ90, Laz03]) in two steps. Our purpose is to distinguish between the part of the structure that comes from the $A_{[0, \infty}$ [-structure (next section) and the part that does not (this section). This will be useful later on when we will transport $A_{[0, \infty[\text {-structures. }}$

Let $k$ be a commutative ring. A graded $k$-quiver is a quiver enriched in the category $G(k)$. More precisely, a graded $k$-quiver $\mathfrak{a}$ consists of a set of objects $\mathrm{Ob}(\mathfrak{a})$ and for $A, A^{\prime} \in \mathrm{Ob}(\mathfrak{a})$, a graded object $\mathfrak{a}\left(A, A^{\prime}\right) \in G(k)$. Since we only use graded $k$-quivers in this paper, we systematically call them simply quivers. The category of quivers with a fixed set of objects admits a tensor product

$$
\mathfrak{a} \otimes \mathfrak{b}\left(A, A^{\prime}\right)=\bigoplus_{A^{\prime \prime}} \mathfrak{a}\left(A^{\prime \prime}, A^{\prime}\right) \otimes \mathfrak{b}\left(A, A^{\prime \prime}\right)
$$

and an internal hom

$$
[\mathfrak{a}, \mathfrak{b}]\left(A, A^{\prime}\right)=\left[\mathfrak{a}\left(A, A^{\prime}\right), \mathfrak{b}\left(A, A^{\prime}\right)\right] .
$$

We put $[\mathfrak{a}, \mathfrak{b}]=\prod_{A, A^{\prime}}[\mathfrak{a}, \mathfrak{b}]\left(A, A^{\prime}\right)$. A morphism of degree $p$ from $\mathfrak{a}$ to $\mathfrak{b}$ is, by definition, an element of $[\mathfrak{a}, \mathfrak{b}]^{p}$. The tensor cocategory $T(\mathfrak{a})$ of a quiver $\mathfrak{a}$ is the quiver

$$
T(\mathfrak{a})=\bigoplus_{n \geqslant 0} \mathfrak{a}^{\otimes n}
$$

equipped with the comultiplication $\Delta: T(\mathfrak{a}) \longrightarrow T(\mathfrak{a}) \otimes T(\mathfrak{a})$ which separates tensors. There are natural notions of morphisms and of coderivations between cocategories and there is a $G(k)$ isomorphism

$$
[T(\mathfrak{a}), \mathfrak{a}] \cong \operatorname{Coder}(T(\mathfrak{a}), T(\mathfrak{a})) .
$$

The object $[T(\mathfrak{a}), \mathfrak{a}]$ is naturally a brace algebra. We recall the definition.

Definition 2.1 (Gerstenhaber and Voronov [GV95]). For $V \in G(k)$, the structure of brace algebra on $V$ consists of the datum of (degree zero) operations

$$
V^{\otimes n+1} \longrightarrow V:\left(x, x_{1}, \ldots x_{n}\right) \longmapsto x\left\{x_{1}, \ldots x_{n}\right\}
$$

satisfying the relation

$$
x\left\{x_{1}, \ldots x_{m}\right\}\left\{y_{1}, \ldots y_{n}\right\}=\sum(-1)^{\alpha} x\left\{y_{1}, \ldots, x_{1}\left\{y_{i_{1}}, \ldots\right\}, y_{j_{1}} \ldots, x_{m}\left\{y_{i_{m}}, \ldots\right\}, y_{j_{m}}, \ldots y_{n}\right\}
$$

where $\alpha=\sum_{k=1}^{m}\left|x_{k}\right| \sum_{l=1}^{i_{k}-1}\left|y_{l}\right|$. The associated Lie bracket of a brace algebra is

$$
\langle x, y\rangle=x\{y\}-(-1)^{|x||y|} y\{x\} .
$$

A brace algebra morphism (between two brace algebras) is a graded morphism preserving all of the individual brace operations.

Proposition 2.2. Let $V$ be a brace algebra. The tensor coalgebra $T(V)$ naturally becomes a (graded) bialgebra with the associative multiplication $M: T(V) \otimes T(V) \longrightarrow T(V)$ defined by the compositions

$$
M_{k, l}: V^{\otimes k} \otimes V^{\otimes l} \longrightarrow T(V) \otimes T(V) \longrightarrow T(V) \longrightarrow V
$$

with

$$
M_{1, l}\left(x ; x_{1}, \ldots x_{l}\right)=x\left\{x_{1}, \ldots, x_{l}\right\}
$$

and all other components equal to zero. The unit for the multiplication is $1 \in k=V^{\otimes 0}$. 
Proof. This is standard (see [GJ94]). A coalgebra morphism $M$ is uniquely determined by the components $M_{k, l}$ and the brace algebra axioms translate into the associativity of $M$.

Put

$$
[T(\mathfrak{a}), \mathfrak{a}]_{n}=\left[\mathfrak{a}^{\otimes n}, \mathfrak{a}\right]=\prod_{A_{0}, \ldots, A_{n} \in \mathfrak{a}}\left[\mathfrak{a}\left(A_{n-1}, A_{n}\right) \otimes \cdots \otimes \mathfrak{a}\left(A_{0}, A_{1}\right), \mathfrak{a}\left(A_{0}, A_{n}\right)\right] .
$$

The brace algebra structure on $[T(\mathfrak{a}), \mathfrak{a}]=\prod_{n \geqslant 0}[T(\mathfrak{a}), \mathfrak{a}]_{n}$ is given by the operations

$$
\left.\left.\left.[T(\mathfrak{a}), \mathfrak{a})]_{n} \otimes[T(\mathfrak{a}), \mathfrak{a})\right]_{n_{1}} \otimes \cdots \otimes[T(\mathfrak{a}), \mathfrak{a})\right]_{n_{k}} \longrightarrow[T(\mathfrak{a}), \mathfrak{a})\right]_{n-k+n_{1}+\cdots+n_{k}}
$$

with

$$
\phi\left\{\phi_{1}, \ldots \phi_{n}\right\}=\sum \phi\left(1 \otimes \cdots \otimes \phi_{1} \otimes 1 \otimes \cdots \otimes \phi_{n} \otimes 1 \otimes \cdots \otimes 1\right) .
$$

The associated Lie bracket corresponds to the commutator of coderivations. We put $B \mathfrak{a}=T(\Sigma \mathfrak{a})$ and $\mathbf{C}_{b r}(\mathfrak{a})=[T(\Sigma \mathfrak{a}), \Sigma \mathfrak{a}]=[B \mathfrak{a}, \Sigma \mathfrak{a}]$. Summarizing, the quiver $\mathfrak{a}$ yields towering layers of (graded) algebraic structure:

(0) the quiver $\mathfrak{a}$, that is, the graded objects $\mathfrak{a}\left(A, A^{\prime}\right)$;

(1) the cocategory $B \mathfrak{a}=T(\Sigma \mathfrak{a})$;

(2) the brace algebra $\mathbf{C}_{b r}(\mathfrak{a})=[B \mathfrak{a}, \Sigma \mathfrak{a}] \cong \operatorname{Coder}(B \mathfrak{a}, B \mathfrak{a})$ which is, in particular, a Lie algebra;

$\left(0^{\prime}\right)$ the associated Hochschild object $\mathbf{C}(\mathfrak{a})=\Sigma^{-1} \mathbf{C}_{b r}(\mathfrak{a})$;

$\left(1^{\prime}\right)$ the bialgebra $T\left(\mathbf{C}_{b r}(\mathfrak{a})\right)=B \mathbf{C}(\mathfrak{a})$.

There is a natural inclusion

$$
\mathbf{C}_{b r}(\mathfrak{a}) \longrightarrow T\left(\mathbf{C}_{b r}(\mathfrak{a})=B \mathbf{C}(\mathfrak{a})\right.
$$

of $(2)$ into $\left(1^{\prime}\right)$.

\subsection{The Hochschild complex of an $\boldsymbol{A}_{[0, \infty[\text {-category }}$}

Definition 2.3 (Getzler and Jones [GJ90]). Let $\mathfrak{a}$ be a quiver. An $A_{[0, \infty}$ [-structure on $\mathfrak{a}$ is an element $b \in \mathbf{C}_{b r}^{1}(\mathfrak{a})$ satisfying

$$
b\{b\}=0 .
$$

The morphisms

$$
b_{n}: \Sigma \mathfrak{a}^{\otimes n} \longrightarrow \Sigma \mathfrak{a}
$$

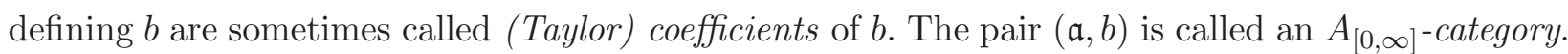
If $b_{0}=0$, it is called an $A_{\infty}$-category. If $b_{n}=0$ for $n \geqslant 3$, it is called a cdg category. If $b_{0}=0$ and $b_{n}=0$ for $n \geqslant 3$, it is called a $d g$ category.

Remark 2.4. Consider $b \in \mathbf{C}_{b r}^{1}(\mathfrak{a})$.

(i) Equation (7) can be written out completely in terms of the coefficients $b_{n}$ of $b$ (see [Lef03, Nic07]).

(ii) If we consider $b$ as a coderivation inside $[B \mathfrak{a}, B \mathfrak{a}]^{1}$, then $(7)$ is equivalent to

$$
b^{2}=b \circ b=0 .
$$

(iii) If we consider $b$ as an element of the bialgebra $B \mathbf{C}(\mathfrak{a})$ through (6), then (7) is equivalent to

$$
b^{2}=M(b, b)=0
$$

where $M$ is the multiplication of $B \mathbf{C}(\mathfrak{a})$.

The easiest morphisms to consider between $A_{[0, \infty}$ [-categories are those with a fixed set of objects. To capture more general morphisms, one could follow the approach of [Lef03] for $A_{\infty}$-categories. 


\section{W. LOWEN}

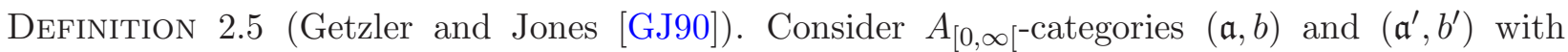
$\mathrm{Ob}(\mathfrak{a})=\mathrm{Ob}\left(\mathfrak{a}^{\prime}\right)$. A (fixed object) morphism of $A_{[0, \infty[}{ }^{- \text {categories }}$ is a (fixed object) morphism of differential graded cocategories $f: B \mathfrak{a} \longrightarrow B \mathfrak{a}^{\prime}$ (that is, a morphism of quivers preserving the comultiplication and the differential). It is determined by morphisms

$$
f_{n}:(\Sigma \mathfrak{a})^{\otimes n} \longrightarrow \Sigma \mathfrak{a}^{\prime}
$$

for $n \geqslant 0$.

An $A_{[0, \infty}$ [-structure on $\mathfrak{a}$ introduces a load of additional algebraic structure on the tower of $\S 2.2$. The Hochschild differential on $\mathbf{C}_{b r}(\mathfrak{a})$ associated to $b$ is given by

$$
d=\langle b,-\rangle \in\left[\mathbf{C}_{b r}(\mathfrak{a}), \mathbf{C}_{b r}(\mathfrak{a})\right]^{1}: \phi \longmapsto\langle b, \phi\rangle
$$

and makes $\mathbf{C}_{b r}(\mathfrak{a})$ into a dg Lie algebra. The complex $\Sigma^{-1} \mathbf{C}_{b r}(\mathfrak{a})$ is (isomorphic to) the classical Hochschild complex of $\mathfrak{a}$. Similarly, considering $b \in B \mathbf{C}(\mathfrak{a})^{1}$, we define a differential

$$
D=[b,-]_{M} \in[B \mathbf{C}(\mathfrak{a}), B \mathbf{C}(\mathfrak{a})]^{1}: \phi \longmapsto[b, \phi]_{M}
$$

where $[-,-]_{M}$ denotes the commutator of the multiplication $M$ determined by the brace operations.

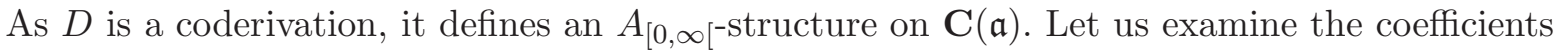

$$
D_{n}: \Sigma \mathbf{C}(\mathfrak{a})^{\otimes n} \longrightarrow \Sigma \mathbf{C}(\mathfrak{a})
$$

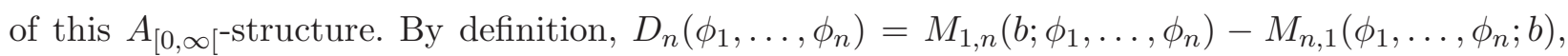
so for $n=1$ we have

$$
D_{1}(\phi)=\langle b, \phi\rangle
$$

whereas for $n>1$ we have

$$
D_{n}\left(\phi_{1}, \ldots, \phi_{n}\right)=b\left\{\phi_{1}, \ldots, \phi_{n}\right\} .
$$

The differential $D$ makes $B \mathbf{C}(\mathfrak{a})$ into a dg bialgebra. By definition, this makes $\mathbf{C}(\mathfrak{a})$ into a $B_{\infty}$ algebra [GJ94]. Summarizing, we obtain the following tower:

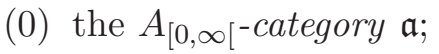

(1) the $d g$ cocategory $B \mathfrak{a}=T(\Sigma \mathfrak{a})$;

(2) the $B_{\infty}$-algebra $\mathbf{C}_{b r}(\mathfrak{a})=[B \mathfrak{a}, \Sigma \mathfrak{a}] \cong \operatorname{Coder}(B \mathfrak{a}, B \mathfrak{a})$ which is, in particular, a $d g$ Lie algebra;

$\left(0^{\prime}\right)$ the associated Hochschild complex $\mathbf{C}(\mathfrak{a})=\Sigma^{-1} \mathbf{C}_{b r}(\mathfrak{a})$;

$\left(1^{\prime}\right)$ the dg bialgebra $T\left(\mathbf{C}_{b r}(\mathfrak{a})\right)=B \mathbf{C}(\mathfrak{a})$.

By a $B_{\infty}$-morphism (between $B_{\infty}$-algebras $B_{1}$ and $B_{2}$ ) we always mean a graded morphism (super)commuting with all of the individual operations on $B_{1}$ and $B_{2}$. A $B_{\infty}$-morphism is a brace algebra morphism, and a very particular case of a morphism of $A_{\infty}$-algebras.

\subsection{Limited functoriality}

The following tautological proposition will be used later on to transfer Hochschild cochains.

Proposition 2.6. Consider quivers $\mathfrak{a}$ and $\mathfrak{b}$ and a brace algebra morphism $\Psi: \mathbf{C}_{b r}(\mathfrak{a}) \longrightarrow \mathbf{C}_{b r}(\mathfrak{b})$.

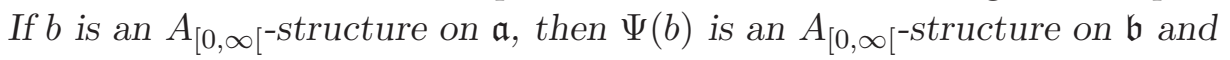

$$
\Psi: \mathbf{C}_{b r}(\mathfrak{a}, b) \longrightarrow \mathbf{C}_{b r}(\mathfrak{b}, \Psi(b))
$$

is a $B_{\infty}$-morphism.

Proof. For $\phi \in T\left(\mathbf{C}_{b r}(\mathfrak{a}, b)\right)$, we show that $T(\Psi)([b, \phi])=[\Psi(b), T(\Psi)(\phi)]$, which immediately follows from the fact that $\Psi$ preserves the brace multiplication. 
Let $\mathfrak{b} \subset \mathfrak{a}$ be the inclusion of a full subquiver, that is, $\mathrm{Ob}(\mathfrak{b}) \subset \mathrm{Ob}(\mathfrak{a})$ and $\mathfrak{b}\left(B, B^{\prime}\right)=\mathfrak{a}\left(B, B^{\prime}\right)$. Using the induced $B \mathfrak{b} \longrightarrow B \mathfrak{a}$, there is a canonical restriction brace algebra morphism

$$
\pi_{\mathfrak{b}}: \mathbf{C}_{b r}(\mathfrak{a}) \longrightarrow \mathbf{C}_{b r}(\mathfrak{b}) .
$$

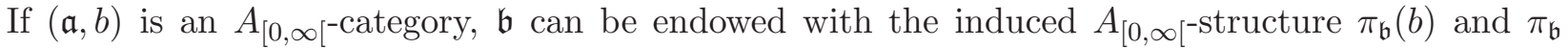
becomes a $B_{\infty}$-morphism (see Proposition 2.6). The $A_{[0, \infty}\left[^{- \text {category }}\left(\mathfrak{b}, \pi_{\mathfrak{b}}(b)\right)\right.$ is called a full $A_{\left[0, \infty\left[{ }^{-}\right.\right.}$ subcategory of $\mathfrak{a}$. In particular, for every object $A \in \mathfrak{a},\left(\mathfrak{a}(A, A), \pi_{A}(b)\right)$ is an $A_{[0, \infty[\text {-algebra. }}$

\subsection{Projection on the zero part}

Let $\mathfrak{a}$ be an $A_{[0, \infty}$ [-category. By the zero part of $\mathbf{C}_{b r}(\mathfrak{a})$ we mean

$$
\mathbf{C}_{b r}(\mathfrak{a})_{0}=[T(\Sigma \mathfrak{a}), \Sigma \mathfrak{a}]_{0}=\prod_{A \in \mathfrak{a}} \Sigma \mathfrak{a}(A, A) .
$$

Consider the graded morphisms

$$
\pi_{0}: \mathbf{C}_{b r}(\mathfrak{a}) \longrightarrow \mathbf{C}_{b r}(\mathfrak{a})_{0}
$$

and $\sigma_{0}: \mathbf{C}_{b r}(\mathfrak{a})_{0} \longrightarrow \mathbf{C}_{b r}(\mathfrak{a})$. The morphism

$$
b_{1} \in[\Sigma \mathfrak{a}, \Sigma \mathfrak{a}]^{1}=\prod_{A, A^{\prime} \in \mathfrak{a}}\left[\Sigma \mathfrak{a}\left(A, A^{\prime}\right), \Sigma \mathfrak{a}\left(A, A^{\prime}\right)\right]^{1}
$$

determines degree 1 morphisms $\left(b_{1}\right)_{A}: \Sigma \mathfrak{a}(A, A) \longrightarrow \Sigma \mathfrak{a}(A, A)$ and a product morphism

$$
b_{1}^{\Delta}: \mathbf{C}_{b r}(\mathfrak{a})_{0} \longrightarrow \mathbf{C}_{b r}(\mathfrak{a})_{0} .
$$

Let $d: \mathbf{C}_{b r}(\mathfrak{a}) \longrightarrow \mathbf{C}_{b r}(\mathfrak{a})$ be the Hochschild differential.

Proposition 2.7. We have

$$
b_{1}^{\Delta}=\pi_{0} d \sigma_{0}
$$

In particular, if $\mathfrak{a}$ is an $A_{\infty}$-category, $\pi_{0}:\left(\mathbf{C}_{b r}(\mathfrak{a}), d\right) \longrightarrow\left(\mathbf{C}_{b r}(\mathfrak{a})_{0}, b_{1}^{\Delta}\right)$ is a morphism of differential graded objects.

Proof. For an element $x \in \Sigma \mathfrak{a}(A, A)$, we have $\pi_{A}(d(x))=\langle b, x\rangle=b_{1}\{x\}=b_{1}(x)$.

\subsection{From $\Sigma \mathfrak{a}$ to $\mathfrak{a}$}

The Hochschild complex of $\mathfrak{a}$ and the $B_{\infty}$-structure on $\Sigma \mathbf{C}(\mathfrak{a})$ are often expressed in terms of $\mathfrak{a}$ rather than $\Sigma \mathfrak{a}$. This can be done using the canonical isomorphisms

$$
\begin{gathered}
\Sigma^{1-n}\left[\mathfrak{a}\left(A_{n-1}, A_{n}\right) \otimes \cdots \otimes \mathfrak{a}\left(A_{0}, A_{1}\right), \mathfrak{a}\left(A_{0}, A_{n}\right)\right] \\
\qquad \\
{\left[\Sigma \mathfrak{a}\left(A_{n-1}, A_{n}\right) \otimes \cdots \otimes \Sigma \mathfrak{a}\left(A_{0}, A_{1}\right), \Sigma \mathfrak{a}\left(A_{0}, A_{n}\right)\right]}
\end{gathered}
$$

determined by the conventions of $\S 2.1$, thus introducing a lot of signs. We define the bigraded object $\mathbf{C}(\mathfrak{a})$ by

$$
\mathbf{C}^{i, n}(\mathfrak{a})=\prod_{A_{0}, \ldots A_{n}}\left[\mathfrak{a}\left(A_{n-1}, A_{n}\right) \otimes \cdots \otimes \mathfrak{a}\left(A_{0}, A_{1}\right), \mathfrak{a}\left(A_{0}, A_{n}\right)\right]^{i} .
$$

An element $\phi \in \mathbf{C}^{i, n}$ has degree $|\phi|=i$, arity $\operatorname{ar}(\phi)=n$ and Hochschild degree $\operatorname{deg}(\phi)=i+n$. We put $\mathbf{C}^{p}(\mathfrak{a})=\prod_{i+n=p} \mathbf{C}^{i, n}(\mathfrak{a})$. The $B_{\infty}$-structure of $\mathbf{C}_{b r}(\mathfrak{a})$ is translated in terms of operations on $\mathbf{C}(\mathfrak{a})$ through $(9)$. The complex $\mathbf{C}(\mathfrak{a})$ is also called the Hochschild complex of $\mathfrak{a}$ and its elements are called Hochschild cochains. For a Hochschild cochain $\phi \in \mathbf{C}^{i, n}(\mathfrak{a})$, the corresponding element of $\mathbf{C}_{b r}(\mathfrak{a})$ has

$$
\sigma^{1-n}(\phi)\left(\sigma f_{n}, \ldots, \sigma f_{1}\right)=(-1)^{n i+(n-1)\left|f_{n}\right|+\cdots+\left|f_{2}\right|} \sigma \phi\left(f_{n}, \ldots, f_{1}\right) .
$$




\section{W. LOWEN}

This identification is different from the others used, for example, in [GJ94, Lef03, Nic07]. Nevertheless, it allows us to recover many standard constructions (up to minor modifications). For example, the operation

$$
\operatorname{dot}: \mathbf{C}_{b r}(\mathfrak{a})_{n} \otimes \mathbf{C}_{b r}(\mathfrak{a})_{m} \longrightarrow \mathbf{C}_{b r}(\mathfrak{a})_{n+m-1}:(x, y) \longmapsto x\{y\}
$$

gives rise to the classical 'dot product'

$$
\bullet: \mathbf{C}^{i, n}(\mathfrak{a}) \otimes \mathbf{C}^{j, m}(\mathfrak{a}) \longrightarrow \mathbf{C}^{i+j, n+m-1}
$$

on $\mathbf{C}(\mathfrak{a})$ given by

$$
\phi \bullet \psi=\sum_{k=0}^{n-1}(-1)^{\epsilon} \phi\left(1^{\otimes n-k-1} \otimes \psi \otimes 1^{\otimes k}\right)
$$

where

$$
\epsilon=(\operatorname{deg}(\phi)+k+1)(\operatorname{ar}(\psi)+1) .
$$

In the following, when no confusion arises, we do not distinguish in the notation between the operations on $\mathbf{C}_{b r}(\mathfrak{a})$ and the induced operations on $\mathbf{C}(\mathfrak{a})$. In particular, the brace operations are

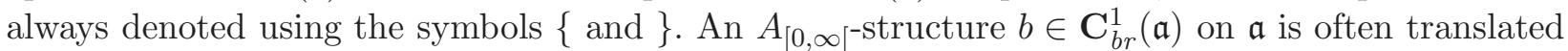

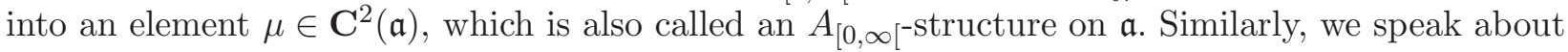
brace algebra and $B_{\infty}$-morphisms between Hochschild complexes $\mathbf{C}(\mathfrak{a}), \mathbf{C}\left(\mathfrak{a}^{\prime}\right)$.

We prove the following result.

Lemma 2.8. Consider $\phi \in \mathbf{C}^{i, n}(\mathfrak{a})$ and $\delta \in \mathbf{C}^{j, 0}(\mathfrak{a})$. We have

$$
\phi\left\{\delta^{\otimes n}\right\}=(-1)^{n(i+((n-1) / 2) j)} \phi(\delta, \ldots, \delta) .
$$

\subsection{The Hochschild complex of a cdg category}

By the previous section a cdg category is a graded quiver $\mathfrak{a}$ together with:

(i) compositions $\mu_{2}=m \in \prod_{A_{0}, A_{1}, A_{2}}\left[\mathfrak{a}\left(A_{1}, A_{2}\right) \otimes \mathfrak{a}\left(A_{0}, A_{1}\right), \mathfrak{a}\left(A_{0}, A_{2}\right)\right]^{0}$;

(ii) differentials $\mu_{1}=d \in \prod_{A_{0}, A_{1}}\left[\mathfrak{a}\left(A_{0}, A_{1}\right), \mathfrak{a}\left(A_{0}, A_{1}\right)\right]^{1}$;

(iii) curvature elements $\mu_{0}=c \in \prod_{A} \mathfrak{a}(A, A)^{2}$;

satisfying the identities:

(i) $d(c)=0$;

(ii) $d^{2}=-m(c \otimes 1-1 \otimes c)$;

(iii) $d m=m(d \otimes 1+1 \otimes d)$;

(iv) $m(m \otimes 1)=m(1 \otimes m)$.

Remark 2.9. Note that identity (ii) differs from the conventional $d^{2}=m(c \otimes 1-1 \otimes c)$ (see [GJ90, Nic07]). However, it suffices to change $c$ into $-c$ to recover the other definition.

Example 2.10. Let $\mathfrak{a}$ be a linear category. An example of a cdg category is the category PCom(a) of precomplexes of $\mathfrak{a}$-objects. A precomplex of $\mathfrak{a}$-objects is a $\mathbb{Z}$-graded $\mathfrak{a}$-object $C$ (with $C^{i} \in \mathfrak{a}$ ) together with a $\mathbb{Z}$-graded a-morphism $\delta_{C}: C \longrightarrow C$ of degree 1 . We have PCom $(\mathfrak{a})(C, D)^{n}=\prod_{i} \mathfrak{a}\left(C^{i}, D^{i+n}\right)$ and, for $f: D \longrightarrow E$ and $g: C \longrightarrow D$ :

(i) $m(f, g)_{i}=(f g)_{i}=f_{i+|g|} g_{i}$,

(ii) $d(f)=\delta_{E} f-(-1)^{|f|} f \delta_{D}$,

(iii) $c_{C}=-\delta_{C}^{2}$. 
Inside PCom(a), we have the usual dg category $\operatorname{Com}(\mathfrak{a})$ of complexes $C$ of a-objects, for which $c_{C}=\delta_{C}^{2}=0$. We use the notation $\mathrm{Com}^{+}(\mathfrak{a})$ and $\mathrm{Com}^{-}(\mathfrak{a})$ for the respective categories of bounded below and bounded above complexes.

As an example of the passage from $\Sigma \mathfrak{a}$ to $\mathfrak{a}$, let us use (10) to compute the Hochschild differential on $\mathbf{C}(\mathfrak{a})$ for a cdg category $\mathfrak{a}$. The differential on $\mathbf{C}_{b r}(\mathfrak{a})$ is given by

$$
\left\langle\sigma c+d+\sigma^{-1} m,-\right\rangle .
$$

Consider $\phi \in \mathbf{C}^{i, n}(\mathfrak{a})$. By definition

$$
\left\langle\sigma c+d+\sigma^{-1} m, \sigma^{1-n} \phi\right\rangle=\operatorname{dot}\left(\sigma c+d+\sigma^{-1} m, \sigma^{1-n} \phi\right)-(-1)^{1-n+i} \operatorname{dot}\left(\sigma^{1-n} \phi, \sigma c+d+\sigma^{-1} m\right) .
$$

The corresponding three terms in terms of $\mathbf{C}(\mathfrak{a})$ are:

(i) $[c, \phi]=c \bullet \phi-(-1)^{\operatorname{deg}(\phi)+1} \phi \bullet c$ which equals

$$
\sum_{k=0}^{n-1}(-1)^{k+1} \phi\left(1^{\otimes n-k-1} \otimes c \otimes 1^{\otimes k}\right)
$$

(ii) $[d, \phi]=d \bullet \phi-(-1)^{\operatorname{deg}(\phi)+1} \phi \bullet d$ which equals

$$
(-1)^{\operatorname{ar} \phi+1}\left(d \phi-(-1)^{|\phi|} \sum_{k=0}^{n-1} \phi\left(1^{\otimes n-k-1} \otimes d \otimes 1^{\otimes k}\right)\right),
$$

(iii) $[m, \phi]=m \bullet \phi-(-1)^{\operatorname{deg}(\phi)+1} \phi \bullet m$ which equals

$$
m(\phi \otimes 1)+\sum_{k=0}^{n-1}(-1)^{k+1} \phi\left(1^{\otimes n-k-1} \otimes m \otimes 1^{\otimes k}\right)+(-1)^{n+1} m(1 \otimes \phi) .
$$

If we look at the bigraded object $\mathbf{C}^{i, n}$ with $i$ being the 'vertical' grading and $n$ being the 'horizontal' grading, then $d_{h}=[m,-]$ defines a horizontal contribution whereas $d_{v}=[d,-]$ defines a vertical contribution to the Hochschild differential $d$. Clearly, up to a factor $(-1)^{n+1}$, the horizontal contribution generalizes the classical Hochschild differential for an associative algebra. If we look at the ' $n$th column' graded object

$$
\mathbf{C}^{*, n}=\prod_{A_{0}, \ldots A_{n}}\left[\mathfrak{a}\left(A_{n-1}, A_{n}\right) \otimes \cdots \otimes \mathfrak{a}\left(A_{0}, A_{1}\right), \mathfrak{a}\left(A_{0}, A_{n}\right)\right]
$$

then the vertical contribution on $\mathbf{C}^{*, n}$ is $(-1)^{n+1}$ times the canonical map induced from $d$. Compared with the $\mathrm{dg}$ case, we have a new curved contribution $d_{c}=[c,-]$ which goes 'two steps up and one step back'. The curved contribution is zero on the zero part $\mathbf{C}^{*, 0}$. In the Hochschild complex of an

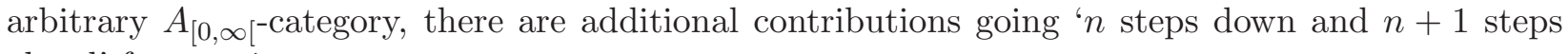
ahead' for $n \geqslant 1$.

\section{A $B_{\infty}$-section to twisted objects}

Let $\mathfrak{a}$ be a quiver. As explained in $\S 2.4$, an inclusion $\mathfrak{a} \subset \mathfrak{a}^{\prime}$ of $\mathfrak{a}$ as a subquiver of some $\mathfrak{a}^{\prime}$ induces a morphism of brace algebras $\pi: \mathbf{C}\left(\mathfrak{a}^{\prime}\right) \longrightarrow \mathbf{C}(\mathfrak{a})$. This section is devoted to the construction of certain quivers $\mathfrak{a}^{\prime}=\operatorname{Tw}(\mathfrak{a})$ of 'twisted objects over $\mathfrak{a}$ ' for which $\pi$ has a certain brace algebra

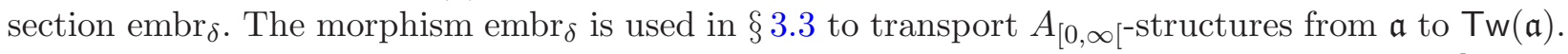
Quivers of twisted complexes encompass the classical twisted complexes over a dg category [BK90, Dri04, Kel99], but also the 'infinite' quivers of semifree dg modules [Dri04] as well as quivers of (pre)complexes over a linear category. The morphism $\mathrm{embr}_{\delta}$ is such that in those examples, it induces the correct $A_{[0, \infty}$ [-structures on these quivers, thus defining a $B_{\infty}$-section of $\pi$. It is used 


\section{W. LOWEN}

in $\S 4.3$ to define the characteristic $d g$ morphism of a linear category $\mathfrak{a}$, which allows us to prove Theorem 4.8 and hence Theorem 1.1. This section is related to ideas in [Fuk03, FOOO, Lef03, Nic07].

\subsection{Some quivers over $\mathfrak{a}$}

Let $\mathfrak{a}$ be a quiver. In this section we define the quiver $\operatorname{Tw}_{\text {free }}(\mathfrak{a})$ of formal coproducts of shifts of $\mathfrak{a}$-objects twisted by a morphism of degree 1. First we define the quiver Free( $\mathfrak{a})$. An object of Free $(\mathfrak{a})$ is a formal expression $M=\bigoplus_{i \in I} \Sigma^{m_{i}} A_{i}$ with $I$ an arbitrary index set, $A_{i} \in \mathfrak{a}$ and $m_{i} \in \mathbb{Z}$. For another $N=\bigoplus_{j \in J} \Sigma^{n_{i}} B_{i} \in$ Free $(\mathfrak{a})$, the graded object Free $(\mathfrak{a})(M, N)$ is, by definition,

$$
\operatorname{Free}(\mathfrak{a})(M, N)=\prod_{i} \bigoplus_{j} \Sigma^{n_{j}-m_{i}} \mathfrak{a}\left(A_{i}, B_{j}\right) .
$$

An element $f \in$ Free $(\mathfrak{a})(M, N)$ can be represented by a matrix $f=\left(f_{j i}\right)$, where $f_{j i}$ represents the element $\sigma^{n_{j}-m_{i}} f_{j i}$.

Definition 3.1. For $M, N$ as above, consider a morphism $f \in \operatorname{Free}(\mathfrak{a})(M, N)$. For a subset $S \subset I$, let $\Phi_{f}(S) \subset J$ be defined by

$$
\Phi_{f}(S)=\left\{j \in J \mid \exists i \in S f_{j i} \neq 0\right\} .
$$

We say that $f \in \operatorname{Free}(M, M)$ is intrinsically locally nilpotent (iln) if for every $i \in I$ there exists $n \in \mathbb{N}$ with $\Phi_{f}^{n}(\{i\})=\emptyset$.

Proposition 3.2. The canonical isomorphisms

$$
\begin{gathered}
{\left[\mathfrak{a}\left(A_{n-1}, A_{n}\right) \otimes \cdots \otimes \mathfrak{a}\left(A_{0}, A_{1}\right), \mathfrak{a}\left(A_{0}, A_{n}\right)\right]} \\
\left.\downarrow \sum^{i_{n}-i_{n-1}} \mathfrak{a}\left(A_{n-1}, A_{n}\right) \otimes \cdots \otimes \sum^{i_{1}-i_{0}} \mathfrak{a}\left(A_{0}, A_{1}\right), \Sigma^{i_{n}-i_{0}} \mathfrak{a}\left(A_{0}, A_{n}\right)\right]
\end{gathered}
$$

for $A_{k} \in \mathfrak{a}, n_{k} \in \mathbb{Z}$ define a morphism of brace algebras

$$
\mathbf{C}(\mathfrak{a}) \longrightarrow \mathbf{C}(\text { Free }(\mathfrak{a})): \phi \longmapsto \phi
$$

with

$$
\phi\left(f_{n}, \ldots, f_{1}\right)_{j i}=\sum_{k_{n-1}, \ldots, k_{1}}(-1)^{\epsilon} \phi\left(\left(f_{n}\right)_{j k_{n-1}},\left(f_{n-1}\right)_{k_{n-1} k_{n-2}}, \ldots,\left(f_{2}\right)_{k_{2} k_{1}},\left(f_{1}\right)_{k_{1} i}\right) .
$$

Lemma 3.3. Consider $\phi \in \mathbf{C}(\mathfrak{a})$ and $\left(f_{n}, \ldots, f_{1}\right) \in \operatorname{Free}(\mathfrak{a})\left(M_{n-1}, M_{n}\right) \otimes \cdots \otimes \operatorname{Free}(\mathfrak{a})\left(M_{0}, M_{1}\right)$. Write $M_{0}=\bigoplus_{i \in I} \Sigma^{\alpha_{i}} A_{i}$ and consider $S \subset I$. There is an inclusion

$$
\Phi_{\phi\left(f_{n}, \ldots, f_{1}\right)}(S) \subset \Phi_{f_{n}}\left(\Phi_{f_{n-1}}\left(\ldots \Phi_{f_{1}}(S)\right)\right) .
$$

Proof. Suppose that $j$ is not contained in the right-hand side. Then for every sequence $j=$ $k_{n}, \ldots, k_{1}, k_{0}=i$ with $i \in S$ one of the entries $\left(f_{p}\right)_{k_{p} k_{p-1}}$ is zero. However, then, looking at the expression (13), clearly $\phi\left(f_{n}, \ldots, f_{1}\right)_{j i}=0$, so $j$ is not contained in the left-hand side.

Next we define the quiver $\operatorname{Tw}_{\text {free }}(\mathfrak{a})$. An object of $\operatorname{Tw}_{\text {free }}(\mathfrak{a})$ is a pair $\left(M, \delta_{M}\right)$ with $M \in \operatorname{Free}(\mathfrak{a})$ and

$$
\delta_{M} \in \operatorname{Free}(\mathfrak{a})(M, M)^{1} .
$$

For $\left(M, \delta_{M}\right),\left(N, \delta_{N}\right) \in \operatorname{Tw}_{\text {free }}(\mathfrak{a}), \operatorname{Tw}_{\text {free }}(\mathfrak{a})\left(\left(M, \delta_{M}\right),\left(N, \delta_{N}\right)\right)=$ Free $(\mathfrak{a})(M, N)$. Consequently, the $\delta_{M}$ determine an element

$$
\delta \in \mathbf{C}^{1}\left(\operatorname{Tw}_{\text {free }}(\mathfrak{a})\right) .
$$

The isomorphisms (11) also define a morphism of brace algebras

$$
\mathbf{C}(\mathfrak{a}) \longrightarrow \mathbf{C}\left(\operatorname{Tw}_{\text {free }}(\mathfrak{a})\right): \phi \longmapsto \phi
$$


which is a section of the canonical projection morphism $\mathbf{C}\left(\operatorname{Tw}_{\text {free }}(\mathfrak{a})\right) \longrightarrow \mathbf{C}(\mathfrak{a})$. In the next section we show that for certain $\mathfrak{a} \subset \mathrm{Tw} \subset \mathrm{T} \mathrm{w}_{\text {free }}$,

$$
\pi: \mathbf{C}(\operatorname{Tw}(\mathfrak{a})) \longrightarrow \mathbf{C}(\mathfrak{a})
$$

has another section depending on $\delta$, which can be used to transport $A_{[0, \infty}$ [-structures. $^{\text {ste }}$

Definition 3.4. A quiver of locally nilpotent twisted objects over $\mathfrak{a}$ is by definition a quiver $\operatorname{Tw}(\mathfrak{a})$ with $\mathfrak{a} \subset \operatorname{Tw}(\mathfrak{a}) \subset \operatorname{Tw}_{\text {free }}(\mathfrak{a})$ such that for every $\phi \in \mathbf{C}(\mathfrak{a})$, for every

$$
\left(f_{n}, \ldots f_{1}\right) \in \operatorname{Tw}(\mathfrak{a})\left(M_{n-1}, M_{n}\right) \otimes \cdots \otimes \operatorname{Tw}(\mathfrak{a})\left(M_{0}, M_{1}\right)
$$

with $M_{0}=\bigoplus_{i \in I} \Sigma^{\alpha_{i}} A_{i}$, and for every $i \in I$ there exists $m_{0} \in \mathbb{N}$ such that for all $m \geqslant m_{0}$, $\Phi_{g}(\{i\})=\emptyset$ for

$$
g=\phi_{m+n}\left\{\delta^{\otimes m}\right\}\left(f_{n}, \ldots f_{1}\right) .
$$

Example 3.5. If $\mathfrak{a}$ is concentrated in degree zero, then $\operatorname{Tw}_{\text {free }}(\mathfrak{a})$ is a quiver of locally nilpotent twisted objects over $\mathfrak{a}$. Indeed, for $\phi \in \mathbf{C}(\mathfrak{a})$, there is only a single $m$ for which the component $\phi_{m}$ is different from zero.

Proposition 3.6. Let $\operatorname{Tw}_{\text {ilnil }}(\mathfrak{a}) \subset \operatorname{Tw}_{\text {free }}(\mathfrak{a})$ be the quiver with as objects the $\left(M, \delta_{M}\right)$ for which $\delta_{M} \in \operatorname{Free}(\mathfrak{a})(M, M)$ is intrinsically locally nilpotent. Then $\mathrm{Tw}_{\text {ilnil }}(\mathfrak{a})$ is a quiver of locally nilpotent twisted objects over $\mathfrak{a}$.

Proof. Consider $\phi,\left(f_{n}, \ldots f_{1}\right)$ and $i$ as in Definition 3.4 and put $\delta_{i}=\delta_{M_{i}}$. For $m \in \mathbb{N}$, consider $g_{m}=\phi_{m+n}\left\{\delta^{\otimes m}\right\}\left(f_{n}, \ldots, f_{1}\right)$. This $g_{m}$ is a sum of expressions

$$
g_{m_{n}, \ldots, m_{0}}=\phi_{m+n}\left(\delta_{n}^{\otimes m_{n}}, f_{n}, \delta_{n-1}^{\otimes m_{n-1}}, \ldots, \delta_{1}^{\otimes m_{1}}, f_{1}, \delta_{0}^{\otimes m_{0}}\right)
$$

with $m_{n}+\cdots+m_{0}=m$. For $\Phi_{g_{m_{n}, \ldots, m_{0}}}(\{i\})$ to be empty, it suffices by Lemma 3.3 that

$$
\Phi_{\delta_{n}}^{m_{n}}\left(\Phi_{f_{n}}\left(\ldots\left(\Phi_{f_{1}}\left(\Phi_{\delta_{0}}^{m_{0}}(\{i\})\right)\right)\right)\right)=\emptyset .
$$

We recursively define numbers $p_{l}$ and finite sets $S_{l}$ for $l=0, \ldots, n$ in the following manner. Put $S_{0}=\{i\}$. Once $S_{l}$ is defined, $p_{l}$ is such that $\Phi_{\delta_{l}}^{p_{l}}\left(S_{l}\right)=\emptyset$ (such a $p_{l}$ exists since $\delta_{l}$ is iln) and $S_{l+1}=\bigcup_{p \in \mathbb{N}} \Phi_{f_{l+1}} \Phi_{\delta_{l}}^{p}\left(S_{l}\right)$. By the pigeonhole principle, if $m \geqslant p_{n}+\cdots+p_{0}$, every $g_{m_{n}, \ldots, m_{0}}$ with $m_{n}+\cdots+m_{0}=m$ has at least one $m_{l} \geqslant p_{l}$, and consequently (16) holds true. Hence, in Definition 3.4, it suffices to take $m_{0}=p_{n}+\cdots+p_{0}$.

\subsection{A word on topology}

Although not strictly necessary, it is convenient to use a bit of topology to understand and reformulate Definition 3.4. The language of this section is used in the proof of Proposition 3.11. All of the topologies we consider will turn the underlying $k$-modules into topological $k$-modules, so in particular we can speak about completions. Put $\mathbf{C}=\mathbf{C}_{b r}(\operatorname{Tw}(\mathfrak{a}))$ for some arbitrary full subcategory $\operatorname{Tw}(\mathfrak{a}) \subset \operatorname{Tw}_{\text {free }}(\mathfrak{a})$. To manipulate certain elements of $B_{\Pi} \mathbf{C}=\prod_{n \geqslant 0}(\Sigma \mathbf{C})^{\otimes n}$ that are not in $B \mathbf{C}$, it will be convenient to consider a certain completion $\hat{B} \mathbf{C}$ of $B \mathbf{C}$. As a first step we endow $B \mathbf{C}$ with a complete Hausdorff 'pointwise' topology $\mathcal{T}_{0}$. To do so we suppose that $\mathfrak{a}$ is naturally a complete Hausdorff topological $k$-quiver, that is, the $\mathfrak{a}\left(A, A^{\prime}\right)$ are complete Hausdorff topological $k$-modules (if there is no natural topology, the $\mathfrak{a}\left(A, A^{\prime}\right)$ are endowed with the discrete topology).

Now consider the algebra multiplication

$$
M: B \mathbf{C} \otimes B \mathbf{C} \longrightarrow B \mathbf{C}
$$

defined by the brace operations. We suppose that $M$ preserves Cauchy nets with respect to $\mathcal{T}_{0}$. For every $\phi \in \mathbf{C}_{b r}(\mathfrak{a}) \subset B \mathbf{C}$ we consider the map

$$
M_{\phi}=M(\phi,-): B \mathbf{C} \longrightarrow\left(B \mathbf{C}, \mathcal{T}_{0}\right) .
$$




\section{W. LOWEN}

Next we endow $B \mathbf{C}$ with the 'weak topology' $\mathcal{T} \subset \mathcal{T}_{0}$ which is by definition the initial topology for the collection $\left(M_{\phi}\right)_{\phi}$, and we let $\hat{B} \mathbf{C}$ denote the completion of $B \mathbf{C}$ with respect to $\mathcal{T}$. The $M_{\phi}$ have natural continuous extensions

$$
\hat{M}_{\phi}: \hat{B} \mathbf{C} \longrightarrow B \mathbf{C} .
$$

Lemma 3.7. For $\psi \in B \mathbf{C}$, the map $M^{\psi}=M(-, \psi): B \mathbf{C} \longrightarrow B \mathbf{C}$ preserves Cauchy nets with respect to $\mathcal{T}$. Consequently, there is a natural continuous extension

$$
\hat{M}^{\psi}: \hat{B} \mathbf{C} \longrightarrow \hat{B} \mathbf{C} \text {. }
$$

Proof. Suppose that we have a $\mathcal{T}$-Cauchy net $\left(x_{\alpha}\right)_{\alpha}$ in $B \mathbf{C}$. We have to show that $M\left(\phi, M\left(x_{\alpha}, \psi\right)\right)$ is $\mathcal{T}_{0}$-Cauchy for every $\phi \in \mathbf{C}_{b r}(\mathfrak{a})$. This follows since $M$ is associative and preserves Cauchy nets.

Definition 3.8. Let $\mathfrak{a}$ be a topological $k$-quiver. A quiver of twisted objects over $\mathfrak{a}$ is by definition a quiver $\mathfrak{a} \subset \operatorname{Tw}(\mathfrak{a}) \subset \operatorname{Tw}_{\text {free }}(\mathfrak{a})$ such that for the canonical $\delta \in \mathbf{C}^{1}(\operatorname{Tw}(\mathfrak{a}))$ the sequence $\left(\sum_{k=0}^{m} \delta^{\otimes k}\right)_{m \geqslant 0}$ converges in $\hat{B} \mathbf{C}$ to a unique element

$$
e^{\delta}=\sum_{k=0}^{\infty} \delta^{\otimes k} .
$$

Remark 3.9. We note that the same suggestive exponential notation is used in [Fuk03].

Proposition 3.10. Let $\mathfrak{a}$ be a $k$-quiver and consider $\mathfrak{a} \subset \operatorname{Tw}(\mathfrak{a}) \subset \operatorname{Tw}_{\text {free }}(\mathfrak{a})$. The following are equivalent:

(i) $\operatorname{Tw}(\mathfrak{a})$ is a quiver of twisted objects over $\mathfrak{a}$ where $\mathfrak{a}$ is endowed with the discrete topology;

(ii) $\operatorname{Tw}(\mathfrak{a})$ is a quiver of locally nilpotent twisted objects over $\mathfrak{a}$.

Proof. By definition of the completion, the sequence converges in $\hat{B} \mathbf{C}$ if and only if for every $\phi \in \mathbf{C}_{b r}(\mathfrak{a})$, the sequence $\left.\left(\sum_{k=0}^{m} \phi\left\{\delta^{\otimes k}\right\}\right)_{m \geqslant 0}\right)$ converges for the 'pointwise discrete' topology $\mathcal{T}_{0}$ on $B$ C. By definition of this topology, this means that for every $\left(f_{n}, \ldots, f_{1}\right)$ and $i \in I$ as in Definition 3.4, there exists an $m_{0}$ such that the general term $\left(\left(\sum_{k=0}^{m} \phi\left\{\delta^{\otimes k}\right\}\left(f_{n}, \ldots, f_{1}\right)(i)\right)\right.$ becomes constant for $m \geqslant m_{0}$. This is clearly equivalent to the fact that the expressions $\phi\left\{\delta^{\otimes k}\right\}\left(f_{n}, \ldots, f_{1}\right)(i)$ become zero for $k \geqslant m_{0}$.

\subsection{Transport of $\boldsymbol{A}_{[0, \infty[\text {-structures to } \mathrm{Tw}(\mathfrak{a})}$}

Let $\mathfrak{a}$ be a topological quiver and consider the inclusion $\mathfrak{a} \subset \operatorname{Tw}(\mathfrak{a})$ of $\mathfrak{a}$ into a quiver of twisted objects over $\mathfrak{a}$ (in particular, $\operatorname{Tw}(\mathfrak{a})$ can be a quiver of locally nilpotent twisted objects over an arbitrary quiver $\mathfrak{a})$. Let

$$
\delta \in \mathbf{C}^{1}(\operatorname{Tw}(\mathfrak{a}))
$$

be the canonical Hochschild cochain of $\operatorname{Tw}(\mathfrak{a})$.

Proposition 3.11. The canonical projection $\pi: \mathbf{C}(\operatorname{Tw}(\mathfrak{a})) \longrightarrow \mathbf{C}(\mathfrak{a})$ has a brace algebra section

$$
\operatorname{embr}_{\delta}: \mathbf{C}(\mathfrak{a}) \longrightarrow \mathbf{C}(\operatorname{Tw}(\mathfrak{a})): \phi \longmapsto \sum_{m=0}^{\infty} \phi\left\{\delta^{\otimes m}\right\}
$$

For $M=\left(M, \delta_{M}\right) \in \operatorname{Tw}(\mathfrak{a})$ and $\phi \in \mathbf{C}^{p}(\mathfrak{a})$, the component $\phi_{M}=\left(\operatorname{embr}_{\delta}(\phi)\right)_{M} \in \operatorname{Tw}(\mathfrak{a})(M, M)^{1}$ is given by

$$
\phi_{M}=\sum_{m=0}^{\infty}(-1)^{\alpha} \phi_{m}\left(\delta_{M}^{\otimes m}\right)
$$

with $\alpha=m((p-m)+(m-1) / 2)$. 
Remark 3.12. If $\operatorname{char}(k)=0$, the map $\operatorname{embr}_{\delta}$ is the Lie morphism $e^{[-, \delta]}$.

Proof. According to Definition 3.8, we dispose of an element $e^{\delta}=\sum_{k=0}^{\infty} \delta^{\otimes k} \in \hat{B} \mathbf{C}$. We define embr $\delta$ to be the restriction of the morphism

$$
\hat{M}\left(-, e^{\delta}\right): B \mathbf{C}(\mathfrak{a}) \longrightarrow B \mathbf{C}(\operatorname{Tw}(\mathfrak{a}))
$$

which exists by $\S 3.2$. In particular, the right-hand side of (19) should be read as a pointwise series, that is, for $\left(f_{n}, \ldots, f_{1}\right) \in \operatorname{Tw}(\mathfrak{a})\left(M_{n-1}, M_{n}\right) \otimes \cdots \otimes \operatorname{Tw}(\mathfrak{a})\left(M_{0}, M_{1}\right)$ where $M_{0}=\bigoplus_{i \in I} \Sigma^{\alpha_{i}} A_{i}$ and $M_{n}=\bigoplus_{j \in J} \Sigma^{\beta_{j}} B_{j}$, we have

$$
\left(\left(\sum_{m=0}^{\infty} \phi\left\{\delta^{\otimes m}\right\}\right)\left(f_{n}, \ldots, f_{1}\right)\right)_{j i}=\sum_{m=0}^{\infty}\left(\left(\phi\left\{\delta^{\otimes m}\right\}\left(f_{n}, \ldots, f_{1}\right)\right)_{j i}\right)
$$

and the right-hand side converges for the topology of $\mathfrak{a}$. Next we verify that (21) is a morphism of algebras, that is, preserves the multiplication $M$. Consider $\phi, \psi \in B \mathbf{C}_{b r}(\mathfrak{a})$. We have

$$
\hat{M}\left(\hat{M}(\phi, \psi), e^{\delta}\right)=\hat{M}\left(\phi, \hat{M}\left(\psi, e^{\delta}\right)\right)=\hat{M}\left(\hat{M}\left(\phi, e^{\delta}\right), \hat{M}\left(\psi, e^{\delta}\right)\right)
$$

where we used the associativity of $M$, continuity of (17) and (18) and the fact that $\hat{M}\left(e^{\delta}, \psi\right)=\psi$. Finally, the statement (20) follows from Lemma 2.8.

Combining Proposition 3.11 with Proposition 2.6, we obtain the following result.

Proposition 3.13. We have the following.

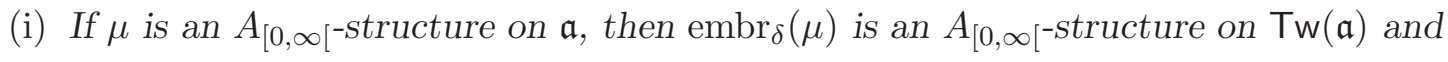

$$
\operatorname{embr}_{\delta}:(\mathfrak{a}, \mu) \longrightarrow\left(\operatorname{Tw}(\mathfrak{a}), \operatorname{embr}_{\delta}(\mu)\right)
$$

is a $B_{\infty}$-morphism.

(ii) If $\mu=c+d+m$ is a cdg structure on $\mathfrak{a}$, then

$$
\operatorname{embr}_{\delta}(\mu)=(c+d\{\delta\}+m\{\delta, \delta\})+(d+m\{\delta\})+m
$$

is a cdg structure on $\operatorname{Tw}(\mathfrak{a})$.

(iii) If $\mu=d+m$ is a $d g$ structure on $\mathfrak{a}$ and $\delta \in \mathbf{C}^{1}(\operatorname{Tw}(\mathfrak{a}))$ satisfies

$$
d\{\delta\}+m\{\delta, \delta\}=0
$$

then

$$
\operatorname{embr}_{\delta}(\mu)=(d+m\{\delta\})+m
$$

is a $d g$ structure on $\operatorname{Tw}(\mathfrak{a})$.

From now on, quivers of twisted objects over an $A_{[0, \infty[}$-category $(\mathfrak{a}, \mu)$ will always be endowed with the $A_{[0, \infty}\left[^{- \text {structure }} \operatorname{embr}_{\delta}(\mu)\right.$.

Remark 3.14. A similar kind of 'transport' is used in [Lef03, Section 6] in order to construct $A_{\infty^{-}}$ functor categories.

\subsection{Classical twisted complexes}

We now discuss how some classical categories of twisted complexes fit into the framework of the previous sections.

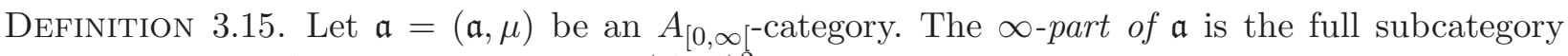
$\mathfrak{a}_{\infty} \subset \mathfrak{a}$ with the $A \in \mathfrak{a}$ for which $\mu_{A} \in \mathfrak{a}(A, A)^{2}$ is zero as objects. 


\section{W. LOWEN}

Example 3.16. Let $\mathfrak{a}$ be an $A_{\infty}$-category and let $\mathrm{tw}_{\text {ilnil }}(\mathfrak{a}) \subset \mathrm{Tw}_{\text {ilnil }}(\mathfrak{a})$ be the quiver with the $\left(M, \delta_{M}\right)$ as objects where $M=\bigoplus_{i=0}^{k} \Sigma^{m_{i}} A_{i}$ is 'finite'.

(i) If $\mathfrak{a}$ is a dg category, then the dg category $\operatorname{tw}_{\text {ilnil }}(\mathfrak{a})_{\infty}$ is equivalent to the classical dg category of twisted complexes over $\mathfrak{a}$ (see [BK90, Dri04, Kel99]). Indeed, the $\infty$-part of $\operatorname{tw}_{\text {ilnil }}(\mathfrak{a})$ is its restriction to the objects $\left(M, \delta_{M}\right)$ with

$$
d\left\{\delta_{M}\right\}+m\left\{\delta_{M}, \delta_{M}\right\}=0 .
$$

More generally, $\operatorname{tw}_{\text {ilnil }}(\mathfrak{a})_{\infty}$ is equivalent to the $A_{\infty}$-category $\operatorname{tw}(\mathfrak{a})$ of twisted objects over $\mathfrak{a}$ (see [Lef03], and [Fuk03] for the algebra case).

(ii) The dg category $T w_{\text {ilnil }}(\mathfrak{a})_{\infty}$ is equivalent to the classical dg category of semifree complexes over $\mathfrak{a}$ (see [Dri04]) which is a dg-model for $D(\mathfrak{a})$, that is, there is an equivalence of triangulated categories $H^{0}\left(\mathrm{Tw}_{\text {ilnil }}(\mathfrak{a})_{\infty}\right) \cong D(\mathfrak{a})$.

Remark 3.17. We conjecture that for an $A_{\infty}$-category a, the $A_{\infty}$-category $\operatorname{Tw}_{\text {ilnil }}(\mathfrak{a})_{\infty}$ is an $A_{\infty^{-}}$ model for the derived category of $\mathfrak{a}$, that is, there is an equivalence of triangulated categories $H^{0}\left(\mathrm{Tw}_{\mathrm{ilnil}}(\mathfrak{a})_{\infty}\right) \cong D_{\infty}(\mathfrak{a})$, where we refer the reader to [Lef03], for definitions of the right-hand side. The finite version of this result has been obtained in [Lef03, Section 7.4].

Remark 3.18. For an $A_{[0, \infty}$ [-category $\mathfrak{a}$, we have $\mathrm{tw}_{\text {ilnil }}(\mathfrak{a})_{\infty}=\operatorname{tw}_{\text {ilnil }}\left(\mathfrak{a}_{\infty}\right)_{\infty}$ and similarly for $\operatorname{Tw}_{\text {ilnil }}(\mathfrak{a})$.

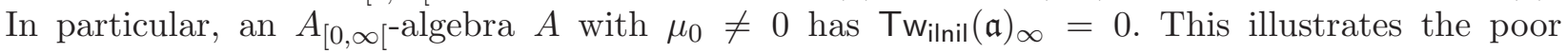
'derivability', in general, of $A_{[0, \infty[\text {-algebras. }}$

The following theorem, which immediately follows from Proposition 3.11, is a refinement of [LV05, Theorem 4.4.1].

Theorem 3.19. Let $\mathfrak{a}$ be a dg category. Then $\operatorname{Tw}(\mathfrak{a})=\operatorname{Tw}$ ilnil $(\mathfrak{a})_{\infty}$ is a dg category which is $d g$ equivalent to the category of semifree dg modules over $\mathfrak{a}$. The canonical projection $\pi: \mathbf{C}(\operatorname{Tw}(\mathfrak{a})) \longrightarrow$ $\mathbf{C}(\mathfrak{a})$ has a $B_{\infty}$-section

$$
\operatorname{embr}_{\delta}: \mathbf{C}(\mathfrak{a}) \longrightarrow \mathbf{C}(\operatorname{Tw}(\mathfrak{a})): \phi \longmapsto \sum_{m=0}^{\infty} \phi\left\{\delta^{\otimes m}\right\}
$$

which is an inverse in the homotopy category of $B_{\infty}$-algebras. In particular, both $\pi$ and $\operatorname{embr}_{\delta}$ are quasi-isomorphisms.

\section{5 (Pre)complexes over linear categories}

Next we apply Proposition 3.11 to categories of (pre)complexes. Let $(\mathfrak{a}, m)$ be a linear category. Consider the quiver $\operatorname{Tw}_{\text {pre }}(\mathfrak{a})$ with

$$
\left(M=\bigoplus_{i \in \mathbb{Z}} \Sigma^{i} A_{i}, \delta_{M}\right)
$$

as objects, with $\delta_{M} \in \operatorname{Free}(\mathfrak{a})(M, M)^{1}$. For another $\left(N=\bigoplus_{i \in \mathbb{Z}} \Sigma^{i} B_{i}, \delta_{N}\right)$, since $\mathfrak{a}$ is concentrated in degree zero, we have $\operatorname{Tw}_{\text {pre }}(\mathfrak{a})(M, N)^{n}=\prod_{i \in \mathbb{Z}} \mathfrak{a}\left(A_{i}, B_{i-n}\right)$. If we change to cohomological notation $A^{i}=A_{-i}$, we have

$$
\operatorname{Tw}_{\text {pre }}(\mathfrak{a})(M, N)^{n}=\prod_{i \in \mathbb{Z}} \mathfrak{a}\left(A^{i}, B^{i+n}\right) .
$$

By Example 3.5, Twpre $(\mathfrak{a})$ is a quiver of locally nilpotent twisted objects over $\mathfrak{a}$. According to Proposition 3.13, the corresponding $A_{[0, \infty}$ [-structure on $\operatorname{Tw}_{\text {pre }}(\mathfrak{a})$ is $\operatorname{embr}_{\delta}(m)=m\{\delta, \delta\}+m\{\delta\}+m$ with

$$
m\{\delta, \delta\}=-\delta^{2}
$$


and

$$
m\{\delta\}=m(\delta \otimes 1-1 \otimes \delta) .
$$

Hence, $T w_{\text {pre }}(\mathfrak{a})$ is precisely the cdg category PCom $(\mathfrak{a})$ of precomplexes of $\mathfrak{a}$-objects of Example 2.10. The category $\operatorname{Tw}_{\text {com }}(\mathfrak{a})=\operatorname{Tw} w_{\text {pre }}(\mathfrak{a})_{\infty}$ is the dg category $\operatorname{Com}(\mathfrak{a})$ of complexes of $\mathfrak{a}$-objects.

Consider the inclusions

$$
\mathfrak{a} \subset \operatorname{Com}^{+}(\mathfrak{a}) \subset \operatorname{Com}(\mathfrak{a}) .
$$

The following result is implicit in [LV05].

Proposition 3.20. The canonical projection $\pi: \mathbf{C}\left(\operatorname{Com}^{+}(\mathfrak{a})\right) \longrightarrow \mathbf{C}(\mathfrak{a})$ is a $B_{\infty}$-quasi-isomorphism.

Proof. Consider the canonical morphisms

$$
\mathfrak{a}^{\mathrm{op}} \longrightarrow \operatorname{Com}^{-}\left(\mathfrak{a}^{\mathrm{op}}\right) \longrightarrow \operatorname{Com}^{-}\left(\operatorname{Mod}\left(\mathfrak{a}^{\mathrm{op}}\right)\right) \longrightarrow \operatorname{Com}\left(\operatorname{Mod}\left(\mathfrak{a}^{\mathrm{op}}\right)\right)=\operatorname{Mod}_{\mathrm{dg}}\left(\mathfrak{a}^{\mathrm{op}}\right) .
$$

A complex in $\operatorname{Com}^{-}\left(\mathfrak{a}^{\text {op }}\right)$ gets mapped to a cofibrant object in $\operatorname{Mod}_{\mathrm{dg}}\left(\mathfrak{a}^{\mathrm{op}}\right)$. Consequently, by [LV05, Theorem 4.4.1], the first map induces a $B_{\infty}$-quasi-isomorphism. The result follows since $\pi$ is induced by the opposite of this map.

Theorem 3.21. The canonical projection $\pi: \mathbf{C}(\mathrm{PCom}(\mathfrak{a})) \longrightarrow \mathbf{C}(\mathfrak{a})$ has a $B_{\infty}$-section

$$
\operatorname{embr}_{\delta}: \mathbf{C}(\mathfrak{a}) \longrightarrow \mathbf{C}(\operatorname{PCom}(\mathfrak{a})): \phi_{n} \longmapsto \sum_{m=0}^{n} \phi_{n}\left\{\delta^{\otimes m}\right\} .
$$

The restrictions of both maps to $\mathbf{C}\left(\mathrm{Com}^{+}(\mathfrak{a})\right)$ are inverse isomorphisms in the homotopy category of $B_{\infty}$-algebras. In particular, they are both quasi-isomorphisms.

\subsection{Abelian categories}

The results of the previous section have an immediate application to abelian categories. Let $\mathcal{A}$ be an abelian category. In [LV05], the Hochschild complex of $\mathcal{A}$ is defined as

$$
\mathbf{C}_{\mathrm{ab}}(\mathcal{A})=\mathbf{C}(\operatorname{lnj}(\operatorname{lnd}(\mathcal{A}))
$$

Let $\mathcal{A}$ be an abelian category with enough injectives and put $\mathfrak{i}=\operatorname{Inj}(\mathcal{A})$. By $[\operatorname{LV} 05$, Theorem 6.6], we have

$$
\mathbf{C}_{\mathrm{ab}}(\mathcal{A}) \cong \mathbf{C}(\mathfrak{i})
$$

and it will be convenient to actually take this as the definition of $\mathbf{C}_{\mathrm{ab}}(\mathcal{A})$.

The dg category $\mathrm{Com}^{+}(\mathfrak{i})$ of bounded below complexes of injectives is a dg model for the bounded below derived category $D^{+}(\mathcal{A})$ of $\mathcal{A}$, hence the notation $D_{\mathrm{dg}}^{+}(\mathcal{A})=\mathrm{Com}^{+}(\mathfrak{i})$. In the spirit of [Kel03], put $\mathbf{C}_{\mathrm{ex}}(\mathcal{A})=\mathbf{C}\left(D_{\mathrm{dg}}^{+}(\mathcal{A})\right)$. With $\mathfrak{a}=\mathfrak{i}$, Theorem 3.21 now yields the following result.

Theorem 3.22. The canonical projection $\pi: \mathbf{C}\left(\mathrm{Com}^{+}(\mathfrak{i})\right) \longrightarrow \mathbf{C}(\mathfrak{i})$ has a $B_{\infty}$-section

$$
\operatorname{embr}_{\delta}: \mathbf{C}(\mathfrak{i}) \longrightarrow \mathbf{C}\left(\mathrm{Com}^{+}(\mathfrak{i})\right): \phi_{n} \longmapsto \sum_{m=0}^{n} \phi_{n}\left\{\delta^{\otimes m}\right\}
$$

which is an inverse in the homotopy category of $B_{\infty}$-algebras. In particular, both $\pi$ and $\operatorname{embr}_{\delta}$ are quasi-isomorphisms establishing $\mathbf{C}_{\mathrm{ab}}(\mathcal{A}) \cong \mathbf{C}_{\mathrm{ex}}(\mathcal{A})$.

\section{Deformations}

This section consists largely of applications of Theorem 3.21. We first recall some facts on deformations and the graded centre enabling us to define, in $\S 4.3$, the characteristic dg morphism of 


\section{W. LOWEN}

a linear category, and to show its relation to deformation theory in Theorem 4.8. The remainder of the chapter is devoted to some applications to deformations of (enhanced) derived categories of abelian categories.

Throughout we focus on first-order deformations, that is, deformations along $k[\epsilon] \longrightarrow k$, since they are in the most direct correspondence with Hochschild cohomology. All definitions can be given for arbitrary deformations, and in the classical setting of an Artin local algebra $R$ over a field $k$ of characteristic zero (with maximal ideal $m$ ), the deformation theory is governed by the Maurer-Cartan equation in the Hochschild complex (tensored by $m$ ).

From now on, $k$ will be a field.

\subsection{Deformations of linear and abelian categories}

The deformation theory of linear and abelian categories was developed in [LV06] as a natural extension of Gerstenhaber's deformation theory of algebras [Ger64]. In this section we recall the main definitions. For a commutative ring $R$, let cat $(R)$ denote the (large) category of $R$-linear categories. The forgetful functor $\operatorname{cat}(k) \longrightarrow \operatorname{cat}(k[\epsilon])$ has the left adjoint

$$
k \otimes_{k[\epsilon]}-: \operatorname{cat}(k[\epsilon]) \longrightarrow \operatorname{cat}(k)
$$

and the right adjoint

$$
\operatorname{Hom}_{k[\epsilon]}(k,-): \operatorname{cat}(k[\epsilon]) \longrightarrow \operatorname{cat}(k)
$$

where $\operatorname{Hom}_{k[\epsilon]}$ denotes the category of $k[\epsilon]$-linear functors. Clearly, for $\mathcal{B} \in \operatorname{cat}(k[\epsilon])$, there is a canonical inclusion functor $\operatorname{Hom}_{k[\epsilon]}(k, \mathcal{B}) \longrightarrow \mathcal{B}$ identifying $\operatorname{Hom}_{k[\epsilon]}(k, \mathcal{B})$ with the full subcategory of objects $B \in \mathcal{B}$ for which $\epsilon: B \longrightarrow B$ is equal to zero.

In [LV06], a notion of flatness for abelian $R$-linear categories is defined which is such that an $R$-linear category $\mathfrak{a}$ is flat (in the sense that it has $R$-flat hom-modules) if and only if the module category $\operatorname{Mod}(\mathfrak{a})$ is flat as an abelian category.

\section{DEFINITION 4.1.}

(i) Let $\mathfrak{a}$ be a $k$-linear category. A first-order linear deformation of $\mathfrak{a}$ is a flat $k[\epsilon]$-linear category $\mathfrak{b}$ together with an isomorphism $k \otimes_{k[\epsilon]} \mathfrak{b} \cong \mathfrak{a}$ in $\operatorname{cat}(k)$.

(ii) Let $\mathcal{A}$ be an abelian $k$-linear category. A first-order abelian deformation of $\mathcal{A}$ is a flat abelian $k[\epsilon]$-linear category $\mathcal{B}$ together with an equivalence of categories $\mathcal{A} \cong \operatorname{Hom}_{k[\epsilon]}(k, \mathcal{B})$.

We denote the natural groupoids of linear deformations of $\mathfrak{a}$ and of abelian deformations of $\mathcal{A}$ by $\operatorname{Def}_{\mathfrak{a}}(k[\epsilon])$ and ab $-\operatorname{Def}_{\mathcal{A}}(k[\epsilon])$, respectively. In [LV06], the notation $\operatorname{def}_{\mathfrak{a}}^{s}$ and $\operatorname{Def}_{\mathcal{A}}$ is used and the terminology strict deformation is used in the linear case.

The following proposition extends the well-known result for algebras.

Proposition 4.2. Let $\mathfrak{a}$ be a $k$-linear category. There is a map

$$
Z^{2} \mathbf{C}(\mathfrak{a}) \longrightarrow \mathrm{Ob}\left(\operatorname{Def}_{\mathfrak{a}}(k[\epsilon])\right)
$$

which induces a bijection

$$
H H^{2}(\mathfrak{a}) \longrightarrow \operatorname{Sk}\left(\operatorname{Def}_{\mathfrak{a}}(k[\epsilon])\right)
$$

Proof. Consider $\phi \in Z^{2} \mathbf{C}(\mathfrak{a})$. The cocycle $\phi$ describes the corresponding linear deformation of $(\mathfrak{a}, m)$ in the following way. Consider the quiver $\mathfrak{a}[\epsilon]=k[\epsilon] \otimes_{k} \mathfrak{a}$ over $k[\epsilon]$. The linear deformation of $\mathfrak{a}$ is $\mathfrak{a}_{\phi}[\epsilon]=(\mathfrak{a}[\epsilon], m+\phi \epsilon)$.

Finally, we mention the following fundamental result of [LV05], where the Hochschild cohomology of the abelian category $\mathcal{A}$ is as defined in $\S 3.6$. 
Proposition 4.3. Let $\mathcal{A}$ be a $k$-linear abelian category. There is a bijection

$$
H H_{a b}^{2}(\mathcal{A}) \longrightarrow \mathrm{ab}-\operatorname{Def}_{\mathcal{A}}(k[\epsilon]) \text {. }
$$

\subsection{The centre of a graded category}

We recall the definition of the centre of a graded category (see also [BF08, Section 3]).

Definition 4.4. Let $\mathfrak{a}$ be a graded category. The centre of $\mathfrak{a}$ is the centre of $\mathfrak{a}$ as a category enriched in $G(k)$, that is,

$$
\mathfrak{Z}(\mathfrak{a})=\operatorname{Hom}\left(1_{\mathfrak{a}}, 1_{\mathfrak{a}}\right)
$$

where $1_{\mathfrak{a}}: \mathfrak{a} \longrightarrow \mathfrak{a}$ is the identity functor and Hom denotes the graded module of graded natural transformations.

Remark 4.5. Explicitly, an element in $\mathfrak{Z}(\mathfrak{a})$ is given by an element $\left(\zeta_{A}\right)_{A} \in \prod_{A \in \mathfrak{a}} \mathfrak{a}(A, A)$ with the naturality property that for all $A, A^{\prime} \in \mathfrak{a}$, the following diagram commutes.

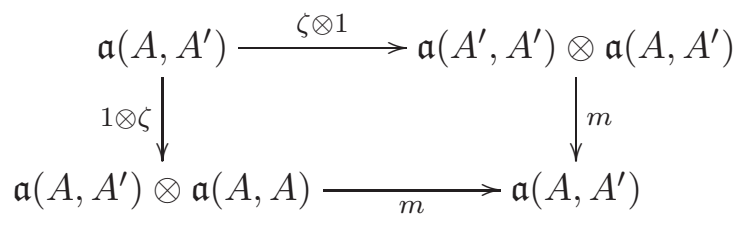

In other words, for $f \in \mathfrak{a}\left(A, A^{\prime}\right)$,

$$
\zeta_{A^{\prime}} f=(-1)^{|f||\zeta|} f \zeta_{A}
$$

Remark 4.6. Let $\mathcal{T}$ be a suspended linear category with suspension $\Sigma_{\mathcal{T}}: \mathcal{T} \longrightarrow \mathcal{T}$. There is an associated graded category $\mathcal{T}_{g r}$ with $\mathcal{T}_{g r}\left(T, T^{\prime}\right)^{n}=\mathcal{T}\left(T, \Sigma_{\mathcal{T}}^{n} T^{\prime}\right)$ and the graded centre of $\mathcal{T}$ is the centre of the graded category $\mathcal{T}_{g r}$. If $\mathfrak{t}$ is an exact $\mathrm{dg}$ category with associated triangulated category $\mathcal{T}=H^{0} \mathfrak{t}$, we have $\mathcal{T}_{g r}=H^{*} \mathfrak{t}$.

\subsection{The characteristic dg morphism}

It is well known that for a $k$-algebra $A$, there is a characteristic morphism of graded commutative algebras from the Hochschild cohomology of $A$ to the graded centre of the derived category $D(A)$. This morphism is determined by the maps, for $M \in D(A)$,

$$
M \otimes{ }_{A}^{L}-: H H_{k}^{*}(A) \cong \operatorname{Ext}_{A^{\mathrm{op}} \otimes A}^{*}(A, A) \longrightarrow \operatorname{Ext}_{A}^{*}(M, M) .
$$

The characteristic morphism occurs, for example, in the theory of support varieties [AB00, EHTSS04, SS04]. Recently, Buchweitz and Flenner proved the existence of a characteristic morphism in the context of morphisms of schemes or analytic spaces [BF08].

In [LV05], it is observed that a characteristic morphism also exists for abelian categories. Let $\mathcal{A}$ be an abelian category with enough injectives, $\mathfrak{i}=\operatorname{Inj}(\mathcal{A})$ and $\operatorname{Com}(\mathfrak{i})$ the dg category of complexes of injectives. As asserted in Proposition 2.7, there is a morphism of differential graded objects

$$
\pi_{0}: \mathbf{C}(\operatorname{Com}(\mathfrak{i})) \longrightarrow \prod_{E \in \operatorname{Com}(\mathfrak{i})} \operatorname{Com}(\mathfrak{i})(E, E)
$$

Taking cohomology of $\pi_{0}$ (where we restrict to $\mathrm{Com}^{+}(\mathfrak{i})$ ) and composing with the isomorphisms $H H_{\mathrm{ab}}^{*}(\mathcal{A}) \cong H H_{\mathrm{ex}}^{*}(\mathcal{A})$ of Theorem 3.22 , we obtain the characteristic morphism

$$
\chi_{\mathcal{A}}: H H_{\mathrm{ab}}^{*}(\mathcal{A}) \longrightarrow \mathfrak{Z}^{*} D^{+}(\mathcal{A}) .
$$

Using the $B_{\infty}$-section of Proposition 3.21, we can actually lift the characteristic morphism to the level of dg objects. In fact, we can construct this lifted characteristic morphism for an arbitrary $k$-linear category $\mathfrak{a}$ instead of $\mathfrak{i}$. 


\section{W. LOWEN}

Definition 4.7. Let $\mathfrak{a}$ be a $k$-linear category. The characteristic $d g$ morphism

$$
\mathbf{C}(\mathfrak{a}) \longrightarrow \prod_{C \in \operatorname{Com}(\mathfrak{a})} \operatorname{Com}(\mathfrak{a})(C, C)
$$

is the composition of the $B_{\infty}$-morphism $\mathbf{C}(\mathfrak{a}) \longrightarrow \mathbf{C}(\operatorname{Com}(\mathfrak{a}))$ of Theorem 3.21 and the projection on the zero part of Proposition 2.7. Taking cohomology, we obtain the characteristic morphism

$$
H H^{*}(\mathfrak{a}) \longrightarrow \mathfrak{Z}^{*} K(\mathfrak{a})
$$

where $K(\mathfrak{a})$ is the homotopy category of complexes of $\mathfrak{a}$-objects.

In the next section we interpret the characteristic morphism in terms of deformation theory.

\subsection{The characteristic morphism and obstructions}

Let $\mathfrak{a}$ be a $k$-linear category. In [Low05], an obstruction theory is established for deforming objects of the homotopy category $K(\mathfrak{a})$. Let $c \in Z^{2} \mathbf{C}(\mathfrak{a})$ be a Hochschild cocycle and $\mathfrak{a}_{c}[\epsilon]$ the corresponding linear deformation. Consider the functor

$$
k \otimes_{k[\epsilon]}-: K\left(\mathfrak{a}_{c}[\epsilon]\right) \longrightarrow K(\mathfrak{a})
$$

and consider $C \in K(\mathfrak{a})$. We say that a (homotopy) c-deformation of $C$ is a lift of $C$ along $k \otimes_{k[\epsilon]}-$. According to [Low05, Theorem 5.2], first-order $c$-deformations of $C$ are governed by an obstruction theory involving $K(\mathfrak{a})(C, C[2])$ and $K(\mathfrak{a})(C, C[1])$. In particular, the obstruction against $c$-deforming $C$ is an element $o_{c} \in K(\mathfrak{a})(C, C[2])$ depending on $c$, whereas $K(\mathfrak{a})(C, C[2])$ itself is independent of $c$. In the remainder of this section we show that the way in which the obstruction $o_{c}$ depends on $c$ is encoded in the characteristic morphism.

Theorem 4.8. Let $\mathfrak{a}$ be a linear category and consider the characteristic morphism

$$
\chi_{\mathfrak{a}}: H H^{2}(\mathfrak{a}) \longrightarrow \mathfrak{Z}^{2} K(\mathfrak{a}) .
$$

We have

$$
\chi_{\mathfrak{a}}(c)=\left(o_{C}\right)_{C \in K(\mathfrak{a})}
$$

where $o_{C} \in K(\mathfrak{a})(C, C[2])$ is the obstruction to $c$-deforming $C$ into an object of $K\left(\mathfrak{a}_{c}[\epsilon]\right)$.

Proof. Let $\bar{\chi}_{\mathfrak{a}}$ be the characteristic dg morphism $\mathbf{C}^{2}(\mathfrak{a}) \longrightarrow \prod_{C \in \operatorname{Com}(\mathfrak{a})} \operatorname{Com}(\mathfrak{a})^{2}(C, C)$ enhancing $\chi_{\mathfrak{a}}$. Consider $C=\left(C, \delta_{C}\right) \in \operatorname{Com}(\mathfrak{a})$ and $\phi \in \mathbf{C}^{2}(\mathfrak{a})$. According to Theorem 3.21, we have

$$
\left(\bar{\chi}_{\mathfrak{a}}(\phi)\right)_{C}=-\phi\left(\delta_{C}, \delta_{C}\right) .
$$

According to [Low05, Theorems 3.8, 4.1], $\left[\phi\left(\delta_{C}, \delta_{C}\right)\right]$ is the obstruction to $c$-deforming $C$.

Corollary 4.9. Let $\mathcal{A}$ be an abelian category with enough injectives. The characteristic morphism

$$
\chi_{\mathcal{A}}: H H_{\mathrm{ab}}^{2}(\mathcal{A}) \longrightarrow \mathfrak{Z}^{2} D^{+}(\mathcal{A})
$$

satisfies

$$
\chi_{\mathcal{A}}(c)=\left(o_{C}\right)_{C \in D^{+}(\mathcal{A})}
$$

where $o_{C} \in \operatorname{Ext}_{\mathcal{A}}^{2}(C, C)$ is the obstruction to deforming $C$ into an object of $D^{+}\left(\mathcal{A}_{c}\right)$. For $C \in D^{b}(\mathcal{A})$, this is equally the obstruction to deforming $C$ into an object of $D^{b}\left(\mathcal{A}_{c}\right)$.

Proof. This easily follows from Theorem 4.8 since $D^{+}(\mathcal{A}) \cong K^{+}(\operatorname{Inj}(\mathcal{A}))$. The last statement follows from [Low05, Section 6.3]. 


\section{5 $A_{[0, \infty[\text {-deformations }}$}

In this section, we discuss the sense in which the Hochschild cohomology of an $A_{\left[0, \infty\left[{ }^{-c a t e g o r y}\right.\right.}$ $\mathfrak{a}$ describes its first-order $A_{[0, \infty}$ [-deformations. The easiest (although not necessarily the best, see Remark 4.17 and $\S 4.7$ ) deformations to handle are those with fixed set of objects. Note that the 'flatness' automatically imposed in this definition is graded freeness, which does not imply cofibrancy in the dg case.

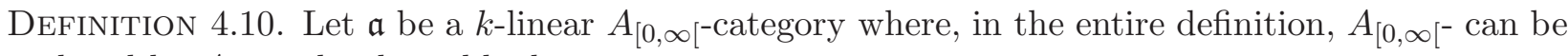
replaced by $A_{\infty^{-}}$, cdg, dg or blank.

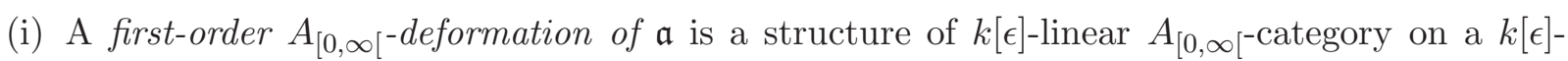
quiver $\mathfrak{b} \cong k[\epsilon] \otimes_{k} \mathfrak{a}$, such that its reduction to $\mathfrak{a}$ coincides with the $A_{[0, \infty}[$-structure of $\mathfrak{a}$ (in other words, the canonical $k \otimes_{k[\epsilon]} \mathfrak{b} \cong \mathfrak{a}$ is an $A_{[0, \infty[\text {-isomorphism). }}$

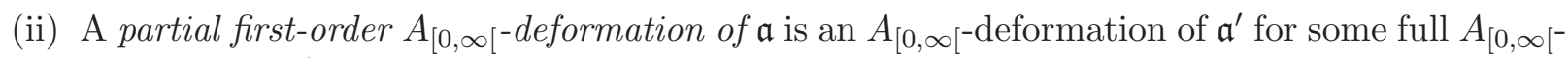
subcategory $\mathfrak{a}^{\prime} \subset \mathfrak{a}$.

(iii) Let $\mathfrak{b}$ and $\mathfrak{b}^{\prime}$ be (partial) deformations of $\mathfrak{a}$. An isomorphism of (partial) deformations is an isomorphism $g: \mathfrak{b} \longrightarrow \mathfrak{b}^{\prime}$ of $A_{[0, \infty}$ [-categories, of which the reduction to $\mathfrak{a}$ (respectively $\mathfrak{a}^{\prime}$ in case of partial deformations) is the identity morphism. A morphism of partial deformations is an isomorphism of deformations between $\mathfrak{b}$ and a full $A_{[0, \infty}$ [-subcategory of $\mathfrak{b}^{\prime}$.

(iv) A partial deformation $\mathfrak{b}$ of $\mathfrak{a}$ is called maximal if every morphism $\mathfrak{b} \longrightarrow \mathfrak{b}^{\prime}$ of partial deformations is an isomorphism.

(v) The groupoid $A_{[0, \infty[}-\operatorname{Def}_{\mathfrak{a}}(k[\epsilon])$ has first-order $A_{[0, \infty[}$-deformations of $\mathfrak{a}$ as objects. Its morphisms are isomorphisms of deformations.

(vi) The category $A_{[0, \infty[}\left[-\operatorname{pDef}_{\mathfrak{a}}(k[\epsilon])\right.$ has first-order partial $A_{[0, \infty}$ [-deformations of $\mathfrak{a}$ as objects. Its morphisms are morphisms of partial deformations.

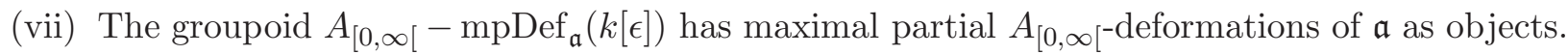
Its morphisms are isomorphisms of partial deformations.

(viii) The groupoid $M C_{\mathfrak{a}}(k[\epsilon])$ has $Z \mathbf{C}^{2}(\mathfrak{a})$ as objects. For $c, c^{\prime} \in Z \mathbf{C}^{2}(\mathfrak{a})$, a morphism $c \longrightarrow c^{\prime}$ is an element $h \in \mathbf{C}^{1}(\mathfrak{a})$ with $d(h)=c^{\prime}-c$.

Proposition 4.11. Let a be a $k$-linear $A_{[0, \infty[\text {-category. }}$

(i) There is an equivalence of categories

$$
M C_{\mathfrak{a}}(k[\epsilon]) \longrightarrow A_{[0, \infty[}-\operatorname{Def}_{\mathfrak{a}}(k[\epsilon]) .
$$

(ii) Consequently, there is a bijection

$$
H H^{2}(\mathfrak{a}) \longrightarrow \operatorname{Sk}\left(A_{[0, \infty[}-\operatorname{Def}_{\mathfrak{a}}(k[\epsilon])\right) .
$$

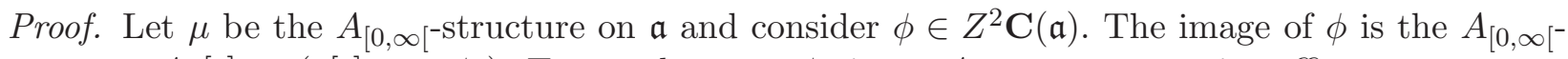
category $A_{\phi}[\epsilon]=(\mathfrak{a}[\epsilon], \mu+\phi \epsilon)$. To see that $\mu+\phi \epsilon$ is an $A_{[0, \infty}$ [-structure, it suffices to compute $\mu+\phi \epsilon\{\mu+\phi \epsilon\}=\mu\{\mu\}+[\mu\{\phi\}+\phi\{\mu\}] \epsilon$ which is zero since $\mu$ is an $A_{[0, \infty}$ [-structure and $\phi$ is a Hochschild cocycle. Next consider a morphism of cocycles $h: \phi \longrightarrow \phi^{\prime}$. The image of $h$ is the morphism $1+h \epsilon: B \mathfrak{a}_{\phi}[\epsilon] \longrightarrow B \mathfrak{a}_{\phi^{\prime}}[\epsilon]$. The identity $d(h)=\phi^{\prime}-\phi$ easily implies the compatibility of $1+h \epsilon$ with the respective $A_{[0, \infty}\left[^{- \text {structures. }}\right.$

Definition 4.12. Consider a $k$-linear $A_{\infty}$-category $\mathfrak{a}$ and $\phi \in H H^{2}(\mathfrak{a})$. The $\phi-\infty$-part of $\mathfrak{a}$ is the full subcategory $\mathfrak{a}_{\phi-\infty} \subset \mathfrak{a}$ with

$$
\mathrm{Ob}\left(\mathfrak{a}_{\phi-\infty}\right)=\left\{A \in \mathfrak{a} \mid 0=H^{2}\left(\pi_{0}\right)(\phi) \in H^{2}(\mathfrak{a}(A, A))\right\}
$$

where $\pi_{0}$ is as in $\S 2.5$. 


\section{W. LOWEN}

Example 4.13. Consider a linear category a and $\phi \in H H^{2}(\mathfrak{a})$. Put $\phi^{\prime}=\left[\operatorname{embr}_{\delta}\right](\phi) \in H H^{2}(\operatorname{Com}(\mathfrak{a}))$. We have

$$
\operatorname{Ob}\left(\operatorname{Com}(\mathfrak{a})_{\phi^{\prime}-\infty}\right)=\left\{C \in \operatorname{Com}(\mathfrak{a}) \mid 0=\chi_{\mathfrak{a}}(\phi)_{C} \in K(\mathfrak{a})(C, C[2])\right\} .
$$

Proposition 4.14. Let $\mathfrak{a}$ be a $k$-linear $A_{\infty}$-category.

(i) There is a morphism

$$
A_{[0, \infty[}-\operatorname{Def}_{\mathfrak{a}}(k[\epsilon]) \longrightarrow A_{\infty}-\operatorname{pDef}_{\mathfrak{a}}(k[\epsilon]): \mathfrak{b} \longmapsto \mathfrak{b}_{\infty}
$$

where $\mathfrak{b}_{\infty}$ is as in Definition 3.15.

(ii) There is a morphism

$$
H H^{2}(\mathfrak{a}) \longrightarrow \operatorname{Sk}\left(A_{\infty}-\operatorname{pDef}_{\mathfrak{a}}(k[\epsilon])\right)
$$

mapping $\phi \in H H^{2}(\mathfrak{a})$ to an $A_{\infty}$-deformation of $\mathfrak{a}_{\phi-\infty} \subset \mathfrak{a}$.

Proof. For part (ii), let $\bar{\phi} \in Z^{2} \mathbf{C}(\mathfrak{a})$ be a Hochschild cocycle with $[\bar{\phi}]=\phi$ and let $\bar{\phi}^{\prime}$ be its restriction to $Z^{2} \mathbf{C}\left(\mathfrak{a}_{\phi-\infty}\right)$. Then $\left(\bar{\phi}^{\prime}\right)_{0} \in \prod_{A \in \mathfrak{a}_{\phi-\infty}} \mathfrak{a}^{2}(A, A)$ is a coboundary, hence there exists $h \in$ $\prod_{A \in \mathfrak{a}_{\phi-\infty}} \mathfrak{a}^{1}(A, A)$ with $d_{\mathfrak{a}}(h)=\left(\bar{\phi}^{\prime}\right)_{0}$. If we consider $h$ as an element of $\mathbf{C}^{1}(\mathfrak{a})$, then $\bar{\phi}^{\prime \prime}=\bar{\phi}^{\prime}-d(h)$ is a representative of $\phi$ with $\left(\bar{\phi}^{\prime}+d(h)\right)_{0}=0$. Consequently, $\left(A_{\phi-\infty}\right)_{\bar{\phi}^{\prime \prime}}[\epsilon]$ is a partial $A_{\infty}$-deformation of $\mathfrak{a}$ corresponding to $\phi$.

\subsection{Deformations of categories of (pre)complexes}

Let $\mathfrak{a}$ be a $k$-linear category. In this section we use Theorem 3.21 to associate to a linear deformation of $\mathfrak{a}$, a cdg deformation of the cdg category PCom( $\mathfrak{a})$ of precomplexes of $\mathfrak{a}$-objects, and a partial dg deformation of the dg category $\operatorname{Com}(\mathfrak{a})$ of complexes of $\mathfrak{a}$-objects.

Combining Theorem 3.21 and Proposition 4.11 we obtain a functor

$$
M C_{\mathfrak{a}}(k[\epsilon]) \longrightarrow M C_{\mathrm{PCom}(\mathfrak{a})}(k[\epsilon]) \longrightarrow A_{[0, \infty[}-\operatorname{Def}_{\mathrm{PCom}(\mathfrak{a})}(k[\epsilon])
$$

factoring through a 'realization' functor

$$
R: M C_{\mathfrak{a}}(k[\epsilon]) \longrightarrow \operatorname{cdg}-\operatorname{Def}_{\mathrm{PCom}(\mathfrak{a})}(k[\epsilon])
$$

whose restriction to $\mathrm{cdg}-\operatorname{Def}_{\mathrm{Com}^{+}(\mathfrak{a})}(k[\epsilon])$ is an equivalence. Similarly, using Proposition 4.14(2), there is a map

$$
\rho^{\prime}: H H^{2}(\mathfrak{a}) \longrightarrow H H^{2}\left(\mathrm{Com}^{+}(\mathfrak{a})\right) \longrightarrow \operatorname{Sk}\left(\mathrm{dg}-\operatorname{pDef}_{\mathrm{Com}^{+}(\mathfrak{a})}(k[\epsilon])\right) .
$$

Theorem 4.15. Consider $\phi \in Z^{2} \mathbf{C}(\mathfrak{a})$ and the corresponding linear deformation $\mathfrak{a}_{\phi}[\epsilon]$.

(i) The cdg deformation $R(\phi)$ of PCom(a) is (isomorphic to) the subcategory PCom triv $\left(\mathfrak{a}_{\phi}[\epsilon]\right) \subset$ $\operatorname{PCom}\left(\mathfrak{a}_{\phi}[\epsilon]\right)$ consisting of the 'trivial' precomplexes $\bar{C}=k[\epsilon] \otimes_{k} C$ for $C \in \operatorname{PCom}(\mathfrak{a})$.

(ii) For every collection of precomplexes $\Gamma=\{\bar{C}\}_{C \in \operatorname{PCom}(\mathfrak{a})}$ where $k \otimes_{k[\epsilon]} \bar{C}=C$, the subcategory $\mathrm{PCom}_{\Gamma}\left(\mathfrak{a}_{\phi}[\epsilon]\right) \subset \mathrm{PCom}\left(\mathfrak{a}_{\phi}[\epsilon]\right)$ spanned by $\Gamma$ is a cdg deformation of $\mathrm{PCom}(\mathfrak{a})$ which is isomorphic to $R(\phi)$.

(iii) For every collection of complexes $\Gamma=\{\bar{C}\}_{C \in \mathrm{Com}^{+}(\mathfrak{a})_{\phi-\infty}}$ where $k \otimes_{k[\epsilon]} \bar{C}=C$, the subcategory $\operatorname{Com}_{\Gamma}^{+}\left(\mathfrak{a}_{\phi}[\epsilon]\right) \subset \operatorname{Com}^{+}\left(\mathfrak{a}_{\phi}[\epsilon]\right)$ spanned by $\Gamma$ is a maximal partial dg deformation of $\operatorname{Com}^{+}(\mathfrak{a})$ representing $\rho^{\prime}([\phi])$.

Consequently, $\rho^{\prime}$ factors over an injection

$$
\rho: H H^{2}(\mathfrak{a}) \longrightarrow \mathrm{Sk}\left(\mathrm{dg}-\operatorname{mpDef}_{\mathrm{Com}^{+}(\mathfrak{a})}(k[\epsilon])\right) .
$$

The image consists of those maximal partial $d g$ deformations that are dg deformations of some $\mathfrak{a}^{\prime}$ with $\mathfrak{a} \subset \mathfrak{a}^{\prime} \subset \mathrm{Com}^{+}(\mathfrak{a})$. 
Remark 4.16. According to Theorem 4.15(iii), the part of $\mathrm{Com}^{+}(\mathfrak{a})$ that 'dg deforms' with respect to $\phi \in H H^{2}(\mathfrak{a})$ is spanned by the objects

$$
\left\{C \in \mathrm{Com}^{+}(\mathfrak{a}) \mid 0=\chi_{\mathfrak{a}}(\phi)_{C} \in K(\mathfrak{a})(C, C[2])\right\} .
$$

Remark 4.17. Morally, Theorem 4.15(ii) suggests that we may consider PCom $\left(\mathfrak{a}_{\phi}[\epsilon]\right)$ as a representative of the class of cdg deformations of PCom $(\mathfrak{a})$ corresponding to the element $[\phi] \in H H^{2}(\mathfrak{a})$. To make this statement mathematically true, one needs a somewhat more relaxed notion of deformation (and isomorphism) in which the object set is not necessarily preserved. Clearly, the statement is true for any such notion of which Definition 4.10 with the isomorphisms relaxed to fully faithful morphisms that are surjective on objects is a special case. In the same spirit, Theorem 4.15(iii) suggests that we may consider $\operatorname{Com}^{+}\left(\mathfrak{a}_{\phi}[\epsilon]\right)$ as a representative of the class of maximal partial $\mathrm{dg}$ deformations of $\mathrm{Com}^{+}(\mathfrak{a})$ corresponding to $[\phi]$.

Proof. There is a canonical morphism of $k[\epsilon]$-quivers

$$
F: \operatorname{PCom}\left(\mathfrak{a}_{\phi}[\epsilon]\right) \longrightarrow k[\epsilon] \otimes_{k} \operatorname{PCom}(\mathfrak{a})
$$

defined in the following manner. A precomplex $\bar{C}$ of $\mathfrak{a}_{\phi}[\epsilon]$-objects is mapped to $C=k \otimes_{k[\epsilon]} \bar{C} \in$ $\operatorname{PCom}(\mathfrak{a})$. For two precomplexes $\bar{C}$ and $\bar{D}, \operatorname{PCom}\left(\mathfrak{a}_{\phi}[\epsilon]\right)^{n}(\bar{C}, \bar{D})=\prod_{i \in \mathbb{Z}} \mathfrak{a}_{\phi}[\epsilon]\left(C^{i}, D^{i+n}\right) \cong k[\epsilon] \otimes$ PCom $(\mathfrak{a})^{n}(C, D)$. This defines $F$. From now on we tacitly use $F$ to identify the left- and the righthand side.

Let us denote the composition of $\mathfrak{a}$ by $m$. By definition, the composition of $\mathfrak{a}_{\phi}[\epsilon]$ is $m+\phi \epsilon$. Write $\delta$ for the predifferentials in PCom $(\mathfrak{a})$ and $\bar{\delta}=\delta+\delta^{\prime} \epsilon$ for the predifferentials in (a full subcategory of) PCom $\left(\mathfrak{a}_{\phi}[\epsilon]\right)$. By Examples 2.10 and 3.5, the cdg structure on PCom(a) is given by $\mu=m\{\delta, \delta\}+$ $m\{\delta\}+m$ and the cdg structure on $\operatorname{PCom}\left(\mathfrak{a}_{\phi}[\epsilon]\right)$ is given by $\tilde{\mu}=(m+\phi \epsilon)\left\{\delta+\delta^{\prime} \epsilon, \delta+\delta^{\prime} \epsilon\right\}+$ $(m+\phi \epsilon)\left\{\delta+\delta^{\prime} \epsilon\right\}+m$. This expression can be rewritten as

$$
\begin{aligned}
& \tilde{\mu}_{0}=m\{\delta, \delta\}+\left[m\left\{\delta, \delta^{\prime}\right\}+m\left\{\delta^{\prime}, \delta\right\}+\phi\{\delta, \delta\}\right] \epsilon, \\
& \tilde{\mu}_{1}=m\{\delta\}+\left[m\left\{\delta^{\prime}\right\}+\phi\{\delta\}\right] \epsilon, \\
& \tilde{\mu}_{2}=m+\phi \epsilon .
\end{aligned}
$$

On the other hand, we have $\operatorname{embr}_{\delta}(\phi)=\phi\{\delta, \delta\}+\phi\{\delta\}+\phi$ so the cdg structure on PCom $(\mathfrak{a})_{\operatorname{embr}_{\delta}(\phi)}[\epsilon]$ is $\bar{\mu}$ with

$$
\begin{aligned}
& \bar{\mu}_{0}=m\{\delta, \delta\}+\phi\{\delta, \delta\} \epsilon, \\
& \bar{\mu}_{1}=m\{\delta\}+\phi\{\delta\} \epsilon, \\
& \bar{\mu}_{2}=m+\phi \epsilon .
\end{aligned}
$$

Comparing $\tilde{\mu}$ and $\bar{\mu}$, it becomes clear that on trivial precomplexes (where $\delta^{\prime}=0$ ), they coincide. This already proves part (i). To produce a deformation isomorphic to $\bar{\mu}$, it is, by Proposition 4.11, allowed to change $\operatorname{embr}_{\delta}(\phi)$ up to a Hochschild coboundary. For a collection $\Gamma$ as in (2), consider the corresponding $\delta^{\prime} \in \mathbf{C}^{1}(\mathrm{PCom}(\mathfrak{a}))$. Using Definition 2.1 and the definition of the Hochschild differential $d$ (see $\S 2.3$ ), it becomes clear that

$$
\tilde{\mu}=\bar{\mu}+d\left(\delta^{\prime}\right) \epsilon
$$

thus proving part (ii).

Now consider a collection $\Gamma$ of complexes as in part (iii). Obviously $\operatorname{Com}_{\Gamma}^{+}\left(\mathfrak{a}_{\phi}[\epsilon]\right)$ defines a dg deformation of $\mathrm{Com}^{+}(\mathfrak{a})_{\phi-\infty}$, hence a partial deformation of $\operatorname{Com}^{+}(\mathfrak{a})$. By the reasoning above, dg deformations of $\mathfrak{a}^{\prime} \subset \mathrm{Com}^{+}(\mathfrak{a})$ isomorphic to $\left.\bar{\mu}\right|_{\mathfrak{a}^{\prime}}$ are precisely given by $\bar{\mu}_{\eta}=\left.\bar{\mu}\right|_{\mathfrak{a}^{\prime}}+d(\eta) \epsilon$ for some $\eta \in \mathbf{C}^{1}\left(\mathfrak{a}^{\prime}\right)$. The existence of an $\eta$ for which $\left(\bar{\mu}_{\eta}\right)_{0}=0$ (and, hence, for which the deformation is $\mathrm{dg}$ ) is equivalent to the existence of $\delta^{\prime} \in \prod_{C \in \mathfrak{a}^{\prime}} \mathfrak{a}^{\prime}(C, C)^{1}$ with $\left(d\left(\delta^{\prime}\right)\right)_{0}=\phi(\delta, \delta)$, in other words 


\section{W. LOWEN}

to the fact that

$$
0=\chi_{\mathfrak{a}}(\phi)_{C} \in H^{2} \operatorname{Com}^{+}(\mathfrak{a})(C, C)
$$

for every $C \in \mathfrak{a}^{\prime}$. Clearly, $\mathfrak{a}^{\prime}=\operatorname{Com}^{+}(\mathfrak{a})_{\phi-\infty}$ is maximal with this property.

Finally, the statement concerning $\rho$ easily follows from the observation that for every $[\phi] \in$ $H H^{2}(\mathfrak{a}), \mathfrak{a} \subset \operatorname{Com}^{+}(\mathfrak{a})_{\phi-\infty}$.

\subsection{Deformations of derived categories}

Let $\mathcal{A}$ be an abelian category with enough injectives. Putting $\mathfrak{a}=\operatorname{lnj}(\mathcal{A})$ in the previous section, we obtain a bijection

$$
\operatorname{Sk}(R): H H_{\mathrm{ab}}^{2}(\mathcal{A}) \longrightarrow \operatorname{Sk}\left(\operatorname{cdg}-\operatorname{Def}_{D_{\mathrm{dg}}^{+}(\mathcal{A})}(k[\epsilon])\right)
$$

and the morphism $\rho^{\prime}$ translates into

$$
\rho^{\prime}: H H_{\mathrm{ab}}^{2}(\mathcal{A}) \longrightarrow \operatorname{Sk}\left(\mathrm{dg}-\mathrm{pDef}_{D_{\mathrm{dg}}^{+}(\mathcal{A})}(k[\epsilon])\right) .
$$

The following now immediately follows from Theorem 4.15 .

Theorem 4.18. Consider $\phi \in Z^{2} \mathbf{C}_{\mathrm{ab}}(\mathcal{A})$ and the corresponding abelian deformation $\mathcal{A}_{\phi}$ of $\mathcal{A}$. Consider the subcategory $D_{\mathrm{dg}}^{+}(\mathcal{A})_{\phi-\infty} \subset D_{\mathrm{dg}}^{+}(\mathcal{A})$ spanned by the complexes $C$ with

$$
0=\chi_{\mathcal{A}}(\phi)_{C} \in D^{+}(\mathcal{A})(C, C[2]) .
$$

For every collection $\Gamma=\{\bar{C}\}_{C \in D_{\mathrm{dg}}^{+}(\mathcal{A})_{\phi-\infty}}$ of bounded below complexes of $\mathcal{A}_{\phi^{-}}$-injectives with $k \otimes_{k[\epsilon]}$ $\bar{C}=C$, the subcategory $D_{\mathrm{dg}, \Gamma}^{+}\left(\mathcal{A}_{\phi}\right) \subset D_{\mathrm{dg}}^{+}\left(\mathcal{A}_{\phi}\right)$ spanned by $\Gamma$ is a dg deformation of $D_{\mathrm{dg}}^{+}(\mathcal{A})_{\phi-\infty}$ and a maximal partial dg deformation of $D_{\mathrm{dg}}^{+}(\mathcal{A})$ representing $\rho^{\prime}([\phi])$. Consequently, $\rho^{\prime}$ factors over an injection

$$
\rho: H H_{\mathrm{ab}}^{2}(\mathcal{A}) \longrightarrow \operatorname{Sk}\left(\mathrm{dg}-\operatorname{mpDef}_{D_{\mathrm{dg}}^{+}\left(\mathcal{A}_{\phi}\right)}(k[\epsilon])\right) .
$$

The image consists of those maximal partial dg deformations that are dg deformations of some $\mathfrak{a}$ with $\operatorname{lnj}(\mathcal{A}) \subset \mathfrak{a} \subset D_{\mathrm{dg}}^{+}(\mathcal{A})$.

Remark 4.19. Theorem 4.18 suggests that we may consider $D_{\mathrm{dg}}^{+}\left(\mathcal{A}_{\phi}\right)$ as a representative of the class of maximal partial dg deformations of $D_{\mathrm{dg}}^{+}(\mathcal{A})$ corresponding to $[\phi]$ (see also Remark 4.17).

Remark 4.20. A 'bounded' version of Theorem 4.18 also holds true: simply replace every dg category $D_{\mathrm{dg}}^{+}$in the theorem by its bounded version $D_{\mathrm{dg}}^{b} \subset D_{\mathrm{dg}}^{+}$spanned by the complexes with bounded cohomology (see also [Low05, Section 6.3]).

As the maps $\operatorname{Sk}(R)$ and $\rho$ are not entirely satisfactory, we propose another sense in which to deform (exact) dg categories, which seems more adapted to (models of) derived categories of abelian categories.

For a commutative ring $R$, let $\operatorname{dgcat}(R)$ denote the (large) category of $R$-linear dg categories. In [Tab05], Tabuada defined a model structure on $\operatorname{dgcat}(R)$ for which the weak equivalences are the quasi-equivalences of $\mathrm{dg}$ categories. Let hodgcat $(R)$ denote the homotopy category for this model structure. In [Toe07], Toën showed that hodgcat $(R)$ is a closed tensor category, with the derived tensor product $\otimes_{R}^{L}$ of dg categories, and with an internal hom between dg categories $\mathfrak{a}$ and $\mathfrak{b}$, which we denote by $\mathcal{R} \mathcal{H o m}_{R}(\mathfrak{a}, \mathfrak{b})$, but which is not a derived version of the internal hom of $\operatorname{dgcat}(R)$ for the above model structure (in fact, it does have a derived interpretation for another model structure defined in $[$ Tab07b]). The forgetful functor hodgcat $(k) \longrightarrow \operatorname{hodgcat}(k[\epsilon])$ has the left adjoint

$$
k \otimes_{k[\epsilon]}^{L}-: \operatorname{hodgcat}(k[\epsilon]) \longrightarrow \operatorname{hodgcat}(k)
$$


and the right adjoint

$$
\mathcal{R H o m}_{k[\epsilon]}(k,-): \operatorname{hodgcat}(k[\epsilon]) \longrightarrow \operatorname{hodgcat}(k) .
$$

Definition 4.21. Let $\mathfrak{a}$ be a $k$-linear dg category.

(i) A first-order homotopy $d g$ deformation of $\mathfrak{a}$ is a $k[\epsilon]$-linear dg category $\mathfrak{b}$ together with an isomorphism $k \otimes_{k[\epsilon]}^{L} \mathfrak{b} \cong \mathfrak{a}$ in $\operatorname{hodgcat}(k)$.

(ii) If $\mathfrak{a}$ is exact, a first-order exact homotopy $d g$ deformation of $\mathfrak{a}$ is a $k[\epsilon]$-linear exact dg category $\mathfrak{b}$ together with an isomorphism $\mathfrak{a} \cong \mathcal{R} \mathcal{H o m}_{k[\epsilon]}(k, \mathfrak{b})$ in hodgcat $(k)$.

Using the techniques of [Toe07], it is not hard to show the following.

Proposition 4.22. Let $\mathcal{A}$ be an abelian $k$-linear category and suppose that $\mathcal{B}$ is a flat abelian deformation of $\mathcal{A}$. Then $D_{\mathrm{dg}}(\mathcal{B})$ is an exact homotopy dg deformation of $D_{\mathrm{dg}}(\mathcal{A})$.

The further investigation of Definition 4.21 (and its variations with respect to other model structures on dg categories [Tab07a, Tab07b, Tab05]) is part of a work in progress.

\section{ACKNOWLEDGEMENTS}

The author is grateful to Bernhard Keller for his continuous interest in, and many pleasant discussions on the topic of this paper. The author acknowledges the hospitality of the Institut de Mathématiques de Jussieu (IMJ) and of the Institut des Hautes Études Scientifiques (IHES) during her postdoctoral fellowship with CNRS.

\section{REFERENCES}

AB00 L. L. Avramov and R.-O. Buchweitz, Support varieties and cohomology over complete intersections, Invent. Math. 142 (2000), 285-318. MR1794064 (2001j:13017).

BLM06 Y. Bespalov, V. Lyubashenko and O. Manzyuk, Closed multicategory of pretriangulated $A_{\infty}$ categories, Preprint (2006), http://www.math.ksu.edu/ lub/papers.html.

BK90 A. I. Bondal and M. M. Kapranov, Framed triangulated categories, Mat. Sb. 181 (1990), 669-683. MR1055981 (91g:18010).

BF08 R.-O. Buchweitz and H. Flenner, Global Hochschild (co-)homology of singular spaces, Adv. Math. 217 (2008), 205-242.

Dri04 V. Drinfeld, DG quotients of DG categories, J. Algebra 272 (2004), 643-691. MR2028075.

EHTSS04 K. Erdmann, M. Holloway, R. Taillefer, N. Snashall and Ø. Solberg, Support varieties for selfinjective algebras, K-Theory 33 (2004), 67-87. MR2199789 (2007f:16014).

Fuk03 K. Fukaya, Deformation theory, homological algebra and mirror symmetry, in Geometry and physics of branes (Como, 2001), Series in High Energy Physics, Cosmology and Gravitation (IOP, Bristol, 2003), pp. 121-209. MR1950958 (2004c:14015).

FOOO K. Fukaya, Y. G. Oh, H. Ohta and K. Ohno, Lagrangian intersection Floer theory-anomaly and obstruction, Preprint, http://www.math.kyoto-u.ac.jp/ fukaya/fukaya.html.

Ger64 M. Gerstenhaber, On the deformation of rings and algebras, Ann. of Math. (2) 79 (1964), 59-103. MR0171807 (30 \#2034).

GV95 M. Gerstenhaber and A. A. Voronov, Homotopy G-algebras and moduli space operad, Int. Math. Res. Notices 3 (1995), 141-153 (electronic). MR1321701 (96c:18004).

GJ90 E. Getzler and J. D. S. Jones, $A_{\infty}$-algebras and the cyclic bar complex, Illinois J. Math. 34 (1990), 256-283. MR1046565 (91e:19001).

GJ94 E. Getzler and J. D. S. Jones, Operads, homotopy algebra and iterated integrals for double loop spaces, Preprint (1994), hep-th/9403055. 
HL04 A. Hamilton and A. Lazarev, Homotopy algebras and noncommutative geometry, Preprint (2004), math.QA/0410621 v1.

Kel03 B. Keller, Derived invariance of higher structures on the Hochschild complex, Preprint (2003), http://www.math.jussieu.fr/ keller/publ/dih.dvi.

Kel99 B. Keller, On the cyclic homology of exact categories, J. Pure Appl. Algebra 136 (1999), 1-56. MR1667558 (99m:18012).

Kel06 B. Keller, A-infinity algebras, modules and functor categories, in Trends in Representation Theory of Algebras and Related Topics, Contemporary Mathematics, vol. 406 (American Mathematical Society, Providence, RI, 2006), pp. 67-93. MR2258042 (2007g:18002).

KS06 M. Kontsevich and Y. Soibelman, Notes on $A_{\infty}$-algebras, $A_{\infty}$-categories and non-commutative geometry I, Preprint (2006), math.RA/0606241v2.

Laz03 A. Lazarev, Hochschild cohomology and moduli spaces of strongly homotopy associative algebras, Homology Homotopy Appl. 5 (2003), 73-100 (electronic). MR1989615 (2004k:16018).

Lef03 K. Lefèvre-Hasegawa, Sur les $A_{\infty}$-catégories, Thèse de doctorat (2003), Université Denis Diderot Paris 7, November, available at the homepage of B. Keller. math.CT/0310337.

Low05 W. Lowen, Obstruction theory for objects in abelian and derived categories, Comm. Algebra 33 (2005), 3195-3223.

LV05 W. Lowen and M. Van den Bergh, Hochschild cohomology of abelian categories and ringed spaces, Adv. Math. 198 (2005), 172-221.

LV06 W. Lowen and M. Van den Bergh, Deformation theory of abelian categories, Trans. Amer. Math. Soc. 358 (2006), 5441-5483.

Lyu03 V. Lyubashenko, Category of $A_{\infty}$-categories, Homology Homotopy Appl. 5 (2003), 1-48 (electronic). MR1989611 (2004e:18008).

Nic07 P. Nicolás, The Bar derived category of a curved dg algebra, Preprint (2007), math.RT/0702449v1.

SS04 N. Snashall and Ø. Solberg, Support varieties and Hochschild cohomology rings, Proc. London Math. Soc. (3) 88 (2004), 705-732. MR2044054 (2005a:16014).

Tab05 G. Tabuada, Une structure de catégorie de modèles de Quillen sur la catégorie des dg-catégories, C. R. Math. Acad. Sci. Paris 340 (2005), 15-19. MR2112034 (2005h:18033).

Tab07a G. Tabuada, Homotopy theory of well-generated algebraic triangulated categories, Preprint (2007), math.KT/0703172v1.

Tab07b G. Tabuada, A new Quillen model for the Morita homotopy theory of $d g$ categories, Preprint (2007), math.KT/0701205v2.

Toe07 B. Toën, The homotopy theory of dg-categories and derived Morita theory, Invent. Math. 167 (2007), 615-667. MR2276263

Wendy Lowen wendy.lowen@ua.ac.be

Department of Mathematics-Informatics, Faculty of Sciences, Universiteit Antwerpen,

Middelheimlaan 1, 2020 Antwerpen, Belgium 